\title{
Multi-periodic photospheric pulsations and connected wind structures in HD 64760*
}

\author{
A. Kaufer ${ }^{1}$, O. Stahl ${ }^{2}$, R. K. Prinja ${ }^{3}$, and D. Witherick ${ }^{3, \star \star ~}$ \\ 1 European Southern Observatory, Alonso de Cordova 3107, Casilla 19001, Santiago 19, Chile \\ e-mail: akaufer@eso.org \\ 2 Landessternwarte Heidelberg, Königstuhl 12, 69117 Heidelberg, Germany \\ 3 Department of Physics \& Astronomy, University College London, Gower Street, London, WC1E 6BT, UK
}

Received 18 July 2005 / Accepted 17 October 2005

\section{ABSTRACT}

\begin{abstract}
We report on the results of an extended optical spectroscopic monitoring campaign on the early-type B supergiant HD 64760 (B0.5 Ib) designed to probe the deep-seated origin of spatial wind structure in massive stars. This new study is based on high-resolution echelle spectra obtained with the FEROS instrument at ESO La Silla. 279 spectra were collected over 10 nights between February 14 and 24, 2003. From the period analysis of the line-profile variability of the photospheric lines we identify three closely spaced periods around $4.810 \mathrm{~h}$ and a splitting of $\pm 3 \%$. The velocity - phase diagrams of the line-profile variations for the distinct periods reveal characteristic prograde non-radial pulsation patterns of high order corresponding to pulsation modes with $l$ and $m$ in the range 6-10. A detailed modeling of the multi-periodic non-radial pulsations with the BRUCE and KYLIE pulsation-model codes (Townsend 1997b, MNRAS, 284, 839) favors either three modes with $l=-m$ and $l=8,6,8$ or $m=-6$ and $l=8,6,10$ with the second case maintaining the closely spaced periods in the co-rotating frame. The pulsation models predict photometric variations of 0.012-0.020 mag consistent with the non-detection of any of the spectroscopic periods by photometry. The three pulsation modes have periods clearly shorter than the characteristic pulsation time scale and show small horizontal velocity fields and hence are identified as p-modes. The beating of the three pulsation modes leads to a retrograde beat pattern with two regions of constructive interference diametrically opposite on the stellar surface and a beat period of $162.8 \mathrm{~h}$ ( 6.8 days). This beat pattern is directly observed in the spectroscopic time series of the photospheric lines. The wind-sensitive lines display features of enhanced emission, which appear to follow the maxima of the photospheric beat pattern. The pulsation models predict for the two regions normalized flux amplitudes of $A=+0.33,-0.28$, sufficiently large to raise spiral co-rotating interaction regions (Cranmer \& Owocki 1996, ApJ, 462, 469). We further investigate the observed H $\alpha$ wind-profile variations with a simple rotating wind model with wind-density modulations to simulate the effect of possible streak lines originating from the localized surface spots created by the NRP beat pattern. It is found that such a simple scenario can explain the time scales and some but not all characteristics of the observed $\mathrm{H} \alpha$ line-profile variations.
\end{abstract}

Key words. stars: early-type - stars: supergiants - stars: individual: HD 64760 - stars: oscillation - stars: mass-loss - stars: rotation

\section{Introduction}

The discovery of systematic, patterned variability in the stellar winds of luminous hot stars rates as one of the major landmarks in recent stellar astrophysics. It is pertinent to our understanding of wind dynamics and stellar structure.

Clearly, to make progress understanding these new phenomena and their impact on the fundamental process of mass-loss via stellar winds - and therefore on stellar evolution scenarios -, the mechanism(s) responsible for changing the starting conditions of the wind and - more specifically - the mechanisms for dividing the stellar surface into distinct regions must be determined. Furthermore, the underlying physical

* Based on observations collected at the European Southern Observatory at La Silla (Proposal IDs 56.D-0235 and 70.D-0433).

$\star \star$ Present address: Physics Department, Trinity College Dublin, Dublin 2, Ireland. mechanisms for coupling the photospheric and wind variations have to be worked out in detail.

The discovery of strictly periodic and sinusoidal modulations of the Si III, Si IV, and N V UV resonance lines with periods of 1.2 and 2.4 days (Prinja et al. 1995; Fullerton et al. 1997) and the recent detection of the same periods in the optical $\mathrm{H} \alpha$ line (Kaufer et al. 2002, KPS 2002 in the following) have turned the intrinsically fast rotating early-type B supergiant HD $64760(\mathrm{~B} 0.5 \mathrm{Ib})$ into a key object in the study of spatially structured hot star winds and their connection to the stellar surface.

KPS 2002 presented the first observational evidence for the physical link between the observed photospheric variations and the large scale wind structure in HD 64760. Photospheric loworder NRP patterns and the constructive or destructive interference of multi-periodic photospheric oscillations were proposed as the most probable source to provide the large-scale 
perturbations at the base of the wind needed to build largescale wind structure. The photospheric UV lines of HD 64760 as observed intensively with IUE were used to probe for the first time the transition zone between the deeper photosphere and the base of the stellar wind by Howarth et al. (1998). Unfortunately, the study was limited by the comparatively low $S / N$ of the IUE spectra. However, this missing link photosphere - wind can now be filled observationally with highest quality optical spectroscopic time series, which is the study presented here.

The optical variability analysis of HD 64760 by KPS 2002 was based on HEROS time series obtained at the ESO $50-\mathrm{cm}$ telescope and suffered from the comparatively low $S / N(\approx 100$ at $\mathrm{H} \alpha$ and dramatically dropping towards the blue) and the poor time resolution ( 1 spectrum per night) of the data set. In particular, the low quality of the spectra inhibited a detailed study of photospheric line-profile variability, which was found to be very subtle, i.e., line-profile variations with peak-to-peak amplitudes of less than $0.5 \%$ of the continuum level.

In this work, we present the results from a follow-up observation campaign with FEROS at the ESO/MPG 2.2-m telescope at La Silla, which provided us with continuous high-quality optical spectroscopic time series over more than two rotational cycles $(\approx 10$ days $)$ with prerequisite high $S / N(\approx 400)$ and spectral $(\approx 50000)$ and time $(\approx 10 \mathrm{~min})$ resolution.

The new data set was acquired with the aim to detect and to determine the characteristics of the photospheric and wind line-profile variations and to further explore the nature of the mechanism that couples them together. The scope of this paper is not to carry out detailed modeling of the observations but to provide detailed input for unified hydrodynamic stellar wind models, which ultimately need to include pulsation (or other physical mechanisms like magnetic fields) as the driver for large scale stellar wind structure.

\section{Observations}

The new observations were carried out with the high-resolution echelle spectrograph FEROS (Kaufer et al. 2000) at the ESO/MPG 2.2-m telescope at La Silla. A total of 279 spectra with a resolving power of $R=48000$ and a wavelength coverage from 3600-9200 $\AA$ were collected over 10 nights between February 14 and 24, 2003. In a typical night, some 30 spectra were recorded over time intervals of $\sim 4 \mathrm{~h}$. Table 1 provides a more detailed record on the distribution of the individual observations over the different nights. The average exposure time per spectrum was $400 \mathrm{~s}$ but varied between $200 \mathrm{~s}$ and $820 \mathrm{~s}$ to account for the variations of the observing conditions. A very high average $S / N$-ratio of 390 per spectrum (at $5400 \AA$ ) was obtained for this time series.

Flatfield and wavelength-calibration exposures have been obtained with the instrument-internal halogen and thoriumargon lamps at the beginning of the respective nights. All spectra have been reduced semi-automatically with ESO-MIDAS using the dedicated FEROS context as described e.g. in Stahl et al. (1999). All spectra have been reduced to barycentric velocities and have been normalized to the stellar continuum using the very stable instrument response curve and low-order fits to clean stellar continuum points.
Table 1. Observation log of the FERos observations of HD 64760 at the ESO/MPG 2.2-m telescope.

\begin{tabular}{cccc}
\hline \hline Night \# & Spectra \# & UTC range & Date \\
\hline 1 & $1-31$ & $00: 38-04: 53$ & $2003-02-15$ \\
2 & $32-64$ & $00: 30-04: 51$ & $2003-02-16$ \\
3 & $65-110$ & $00: 23-07: 47$ & $2003-02-17$ \\
4 & $111-145$ & $00: 21-04: 56$ & $2003-02-18$ \\
5 & $146-172$ & $00: 41-04: 54$ & $2003-02-19$ \\
6 & $173-200$ & $00: 27-04: 54$ & $2003-02-20$ \\
7 & $201-204$ & $00: 14-00: 49$ & $2003-02-21$ \\
8 & $205-224$ & $00: 18-04: 44$ & $2003-02-22$ \\
9 & $225-249$ & $00: 08-04: 43$ & $2003-02-23$ \\
10 & $250-279$ & $00: 31-04: 47$ & $2003-02-24$ \\
\hline
\end{tabular}

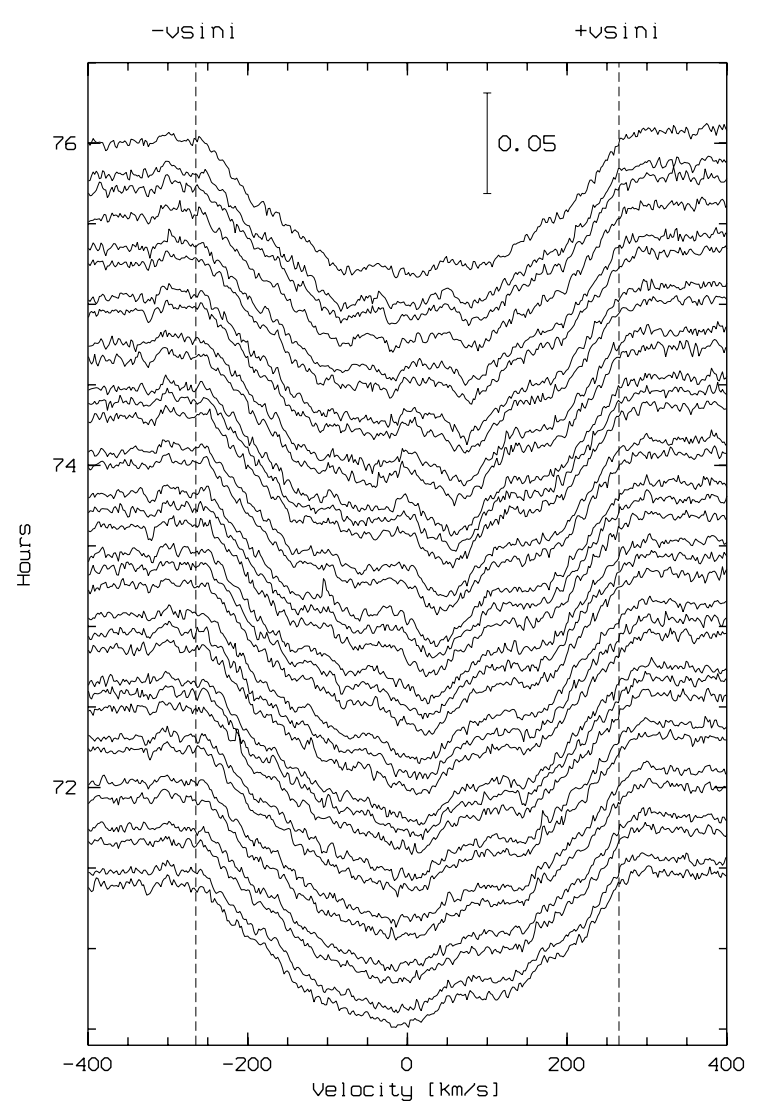

Fig. 1. Time series of spectra of the Si III $\lambda 4553$ line in night 4 of our monitoring campaign. The time axis increases towards the top and is given in hours since MJD $=52685.0265$. The vertical bar indicates $5 \%$ in intensity with respect to the continuum; $v \sin i=$ $\pm 265 \mathrm{~km} \mathrm{~s}^{-1}$ is indicated by vertical dashed lines.

Throughout this paper, all velocities are given with respect to the laboratory wavelengths of the corresponding lines of interest. For this purpose the wavelengths of the respective lines have been corrected by a systemic velocity of $v_{\text {sys }}=+18 \mathrm{~km} \mathrm{~s}^{-1}$ for HD 64760 as was obtained from the fitting of rotationally broadened synthetic line profiles by KPS 2002 (cf. their Fig. 1). The uncertainty in $v_{\text {sys }}$ from the fits amounts to $\pm 2 \mathrm{~km} \mathrm{~s}^{-1}$.

Figure 1 shows an example of the 35 spectra around the Si III $\lambda 4553$ line as obtained in night 4 of the monitoring campaign. Systematic line-profile variability (LPV) is directly discernible and will be discussed in detail below. 


\section{Spectroscopic time series}

In the following we present the spectroscopic times series as so-called dynamic spectra where the time series of spectra is displayed in a two-dimensional velocity (wavelength) - time image with the intensity levels of the individual spectra represented by a grayscale from the low (black) to the high (white) intensity cut level. The intensity levels are computed as the difference with respect to the average spectrum of the times series. Therefore, the discernible absorption (black) and emission (white) features in the dynamical spectra are pseudo-absorption and pseudo-emission features relative to the time-averaged stellar spectrum (gray). The average stellar spectrum is constructed with the weights of the individual spectra according to their respective $S / N$-ratio.

In this paper, we present the time series in a collapsed form, not showing the full time gap from night to night. The time gap remains as a white horizontal bar, while times with no data within the dynamical spectra of one night appear as black horizontal bars. The seventh night contains only four spectra and is completely omitted from the dynamical spectra (but not from the analyses below). The dynamical spectra indicate on the right axis the observing night and on the left the start and end time of the corresponding observations as counted in hours from the start time of the exposure of the first spectrum of the time series, i.e., $t=0 \mathrm{~h}$ equals to MJD $=52685.0265$. In addition, all dynamical spectra indicate the projected rotational velocity of $v \sin i= \pm 265 \mathrm{~km} \mathrm{~s}^{-1}$ as was determined in KPS 2002 for HD 64760.

The dynamical spectra of the representative purely photospheric Si III $\lambda 4553$ line, the photospheric He I $\lambda 6678$ line, which shows some wind emission contribution and the mostly wind-sensitive $\mathrm{H} \alpha$ line are shown in Figs. 2-4, respectively. Virtually all available isolated photospheric lines in the spectrum of HD 64760 in the accessible wavelength range from 3600-9200 ̊ have been examined through their dynamical spectra and are found to appear very similar - but very different from $\mathrm{H} \alpha$. The variations in the photospheric lines are largest in the shown Si III $\lambda 4553$ and He I $\lambda 6678$ lines with peakto-peak amplitudes of $\pm 1 \%$ relative to the time-averaged spectrum. The strong He I $\lambda 6678$ line does show in addition to the photospheric patterns some wind features from $\mathrm{H} \alpha$, e.g., most prominent, a broad pseudo-emission component traveling from the red (day 3) to the blue side (days 6-9) of the line. The wind sensitivity of the He I $\lambda 6678$ line is substantiated by a small amount of asymmetry in the time-averaged line profile and the existence of small emission peaks superimposed to the photospheric line profile at $-298 \mathrm{~km} \mathrm{~s}^{-1}$ and $+330 \mathrm{~km} \mathrm{~s}^{-1}$. The emission peaks reach a maximum intensity of $0.4 \%$ above the continuum and are clear mass-loss indicators for a fast rotating wind as was demonstrated for $\mathrm{H} \alpha$ in KPS 2002. No such wind features are found for the Si III $\lambda 4553$ line supporting its bona fide photospheric origin.

The photospheric lines vary strongly within one night, but also the variability pattern looks different from night to night and changes from periods with low variability (like in nights 1 and 6) to periods with strong patterns (like in nights 3 and 9). This observed pattern variability is indicating either a

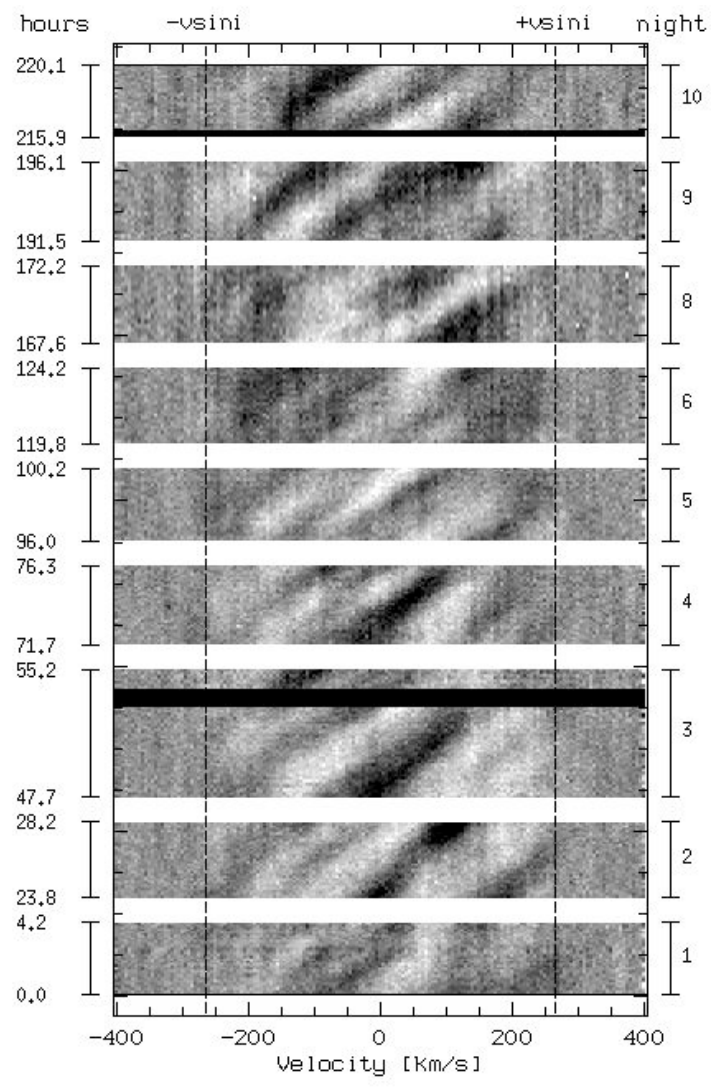

Fig. 2. Dynamical velocity - time spectrum of the Si III $\lambda 4553$ line. On the right axis the observing night and on the left axis the start and end time of the corresponding observations is indicated in hours since $\mathrm{MJD}=52685.0265$. The intensities from black to white are displayed with $\pm 1 \%$ cut levels. Further, $v \sin i$ is indicated by vertical dashed lines.

contribution from non-periodic effects or constructive/ destructive multi-periodic effects. The pseudo-periods become directly discernible if the spectral intensity variations are followed in time for a fixed velocity bin (e.g. at zero-velocity) and appear to be of the order of the length of one observing night (i.e., about 4 to $6 \mathrm{~h}$ ). It is important to note here, that the length of the observing nights was just sufficient to sample the full extent of most of the photospheric variations as can e.g. be seen in Figs. 1 and 2 in night 4 where the pattern at velocity zero evolves from absorption to emission and back to absorption within $4.5 \mathrm{~h}$. At all times, the photospheric lines do clearly show structured variability with alternating prograde traveling pseudo-absorptions and pseudo-emission features in the dynamical spectra, which are indicative of the presence of non-radial pulsations (NRP) in the atmosphere of HD 64760. The fact that the slope of the traveling features appears to be non-constant and that from night to night periods of low and high variability amplitudes can be distinguished favor the presence of multi-periodic NRPs. Baade (1984) reported for the first time photospheric line-profile variations in highestquality optical spectra of the Si III $\lambda 4553$ and He I $\lambda 6678$ lines, which where interpreted as high $(|m|$ large) and low order $(|m| \approx 2)$ NRPs with respective periods of $\sim 0.1$ and $\sim 0.5$ days. Howarth et al. (1998) could detect the 1.2-day period reported 


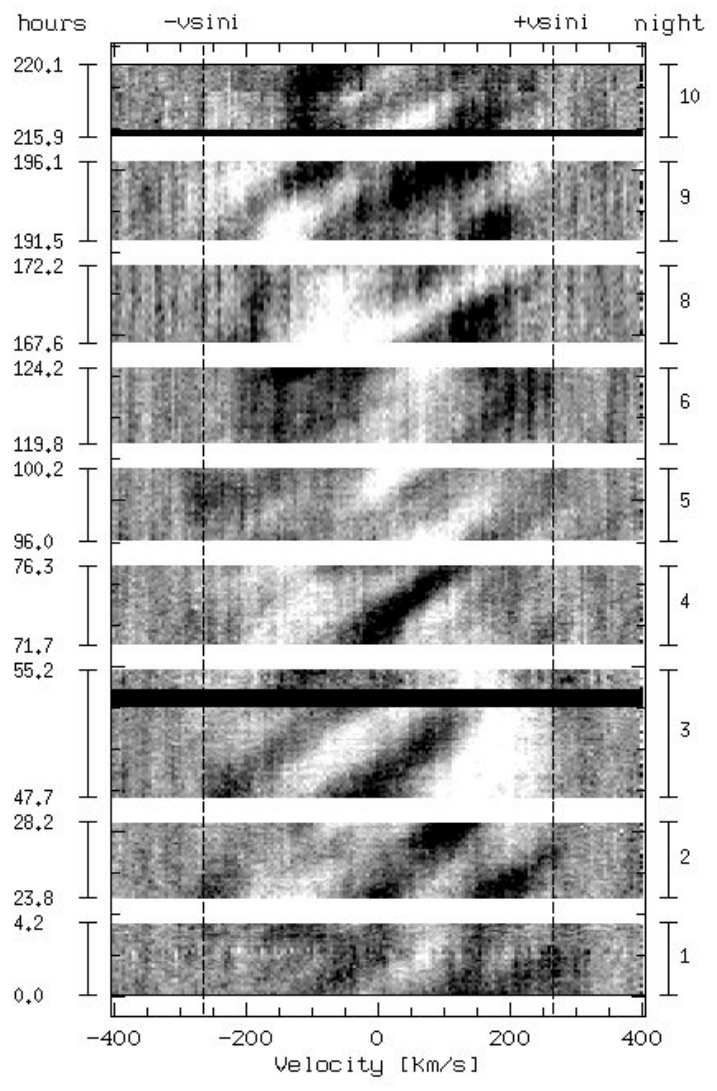

Fig. 3. Dynamical spectrum of the He I $\lambda 6678$ line. Displayed with $\pm 1 \%$ cut levels. $v \sin i$ is indicated by vertical dashed lines.

in the UV wind lines also in the UV photospheric lines of the IUE MEGA data set with a weak signature for a prograde traveling NRP pattern in the corresponding phase diagrams while KPS 2002 report a weak signature of prograde NRPs in the optical He I $\lambda 4026$ line with a 2.4-day period. Only the high quality and proper sampling over several contiguous nights as achieved with this new spectroscopic data set allows for the first time to directly visualize the presence of NRPs in HD 64760. The characterization and modeling of the multiperiodic NRPs in HD 64760 are the main subject of our analysis in Sects. 4 and 5.

In contrast to the photospheric lines the dynamical spectrum of the $\mathrm{H} \alpha$ line as shown in Fig. 4 varies very little within one night, indicating that predominantly longer time scales of the order of days are governing the variability of this line. This finding is consistent with the observations in KPS 2002. However, so far no evidence could be given for any variability on shorter time scales due to the inappropriate 1-day sampling rate in the earlier HEROS data set used in KPS 2002. The new FEROs data set provides for the first time high time resolution for the $\mathrm{H} \alpha$ line together with coverage over several nights. The night-to-night variations in $\mathrm{H} \alpha$ show an evolution of the pseudo-absorption and emission features. At the same time the variations within one night do not reveal the presence of any shorter time scales but are consistent with the line-profile changes as expected from the night-to-night trends. Interestingly an absence of pseudo-absorptions and emissions is noticeable in the nights 1 and 5 almost coincident

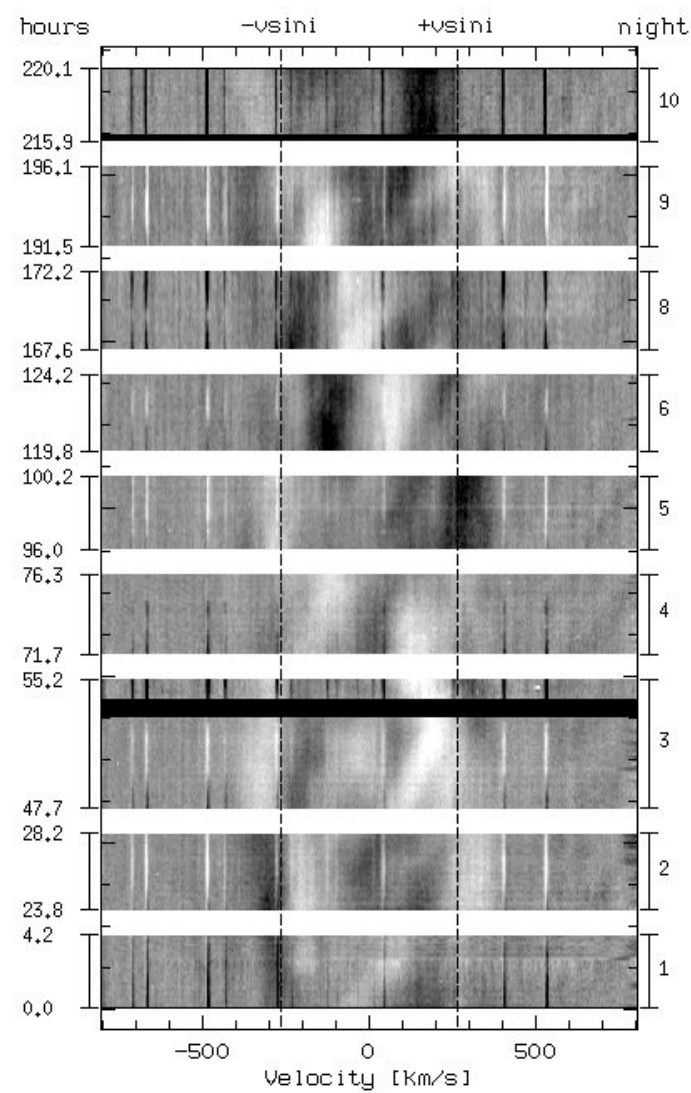

Fig. 4. Dynamical spectrum of the $\mathrm{H} \alpha$ line. Displayed with $\pm 3 \%$ cut levels. $v \sin i$ is indicated by vertical dashed lines. Note the extended velocity range of $\pm 800 \mathrm{~km} \mathrm{~s}^{-1}$ to cover the wind features of the line profile. The sharp spectral features are unresolved telluric lines in the $\mathrm{H} \alpha$ region.

with the low amplitudes of variability in the photospheric lines in the nights 1 and 6 . The $\mathrm{H} \alpha$ line of HD 64760 is strongly affected by the mass loss of the supergiant star and the rotation of the wind as was demonstrated in KPS 2002 (cf. their Fig. 2). However, since their estimate of mass-loss rate of $9 \times 10^{-7} M_{\odot} / \mathrm{yr}$ is still moderate, it is anticipated to find contributions of the underlying photospheric profile and its variability on the shorter time scales as found for all other photospheric lines (cf. above). This is not the case and it is worth noting here, that the absence of NRP patterns in the dynamical spectra of $\mathrm{H} \alpha$ will be "naturally" explained by the NRP modeling as carried out in Sect. 5.

\section{Period analysis}

\subsection{Photospheric lines}

The qualitative findings from the inspection of the dynamical spectra of photospheric and wind lines in Sect. 3 will be quantified in this section by means of a rigorous time-series analysis.

As a first step we carried out a two-dimensional period analysis on the FEROS data set by computing power spectra and periodograms for velocity bins of $5 \mathrm{~km} \mathrm{~s}^{-1}$ width across the lines of interest. The full frequency range sampled by the data set from $1 / 10$ days $^{-1}$ to $1 / 10 \mathrm{~min}^{-1}$ was searched for significant periods by application of the CLEAN algorithm described 


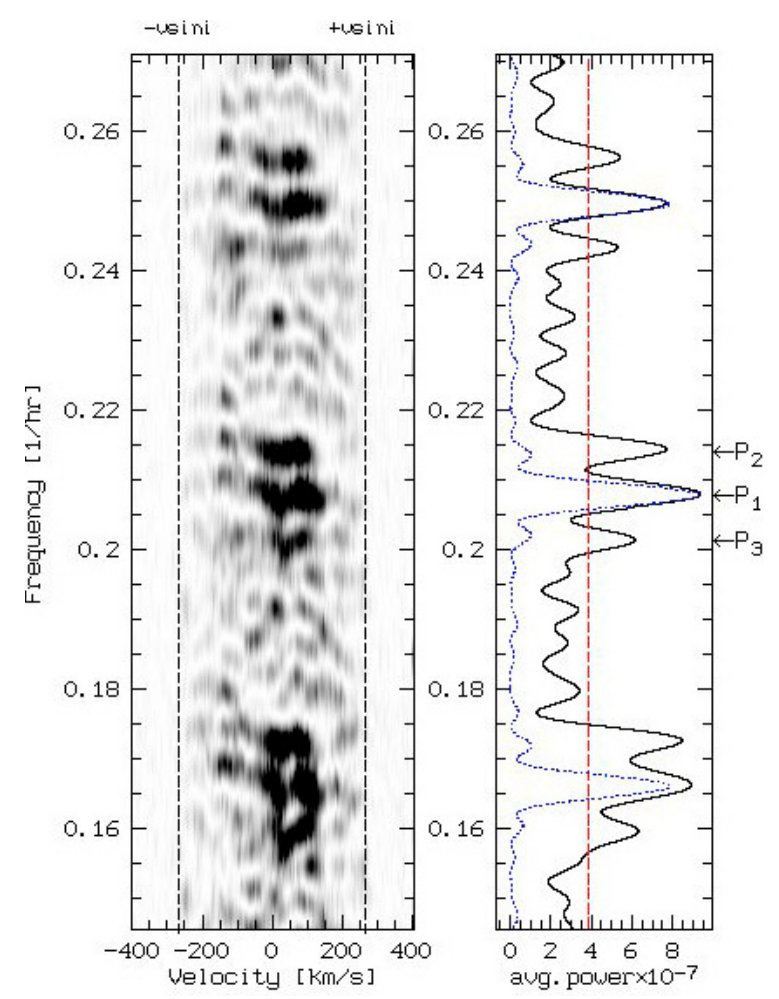

Fig. 5. Two-dimensional power spectrum of the Si III $\lambda 4553$ line around a period of $4.8 \mathrm{~h}$ showing the three significant power peaks as identified by the iterative CLEAN process. The $99.5 \%$ level of significance of the periodogram is indicated by a (red) dashed line. The window function of the data set is shown as (blue) dotted line and was shifted to the position and normalized to the height of the strongest power peak. The strongest alias periods can be seen at $4 \mathrm{~h}$ and $6 \mathrm{~h}$. The three significant periods $P_{1}=4.810 \mathrm{~h}, P_{2}=4.672 \mathrm{~h}, P_{3}=4.967 \mathrm{~h}$ are labeled in the right plot, which displays the average power over the velocity bin of $-v \sin i$ to $+v \sin i$.

by Schwarzenberg-Czerny (1996). This algorithm makes use of the equivalence of the classical CLEAN algorithm and leastsquare fits of sine functions to the observed data (Schwarz 1978) - both performing an iterative deconvolution of the window function from the time series. Periodograms and power spectra are computed using Lomb-Scargle statistics, which take into account for uneven sampling of the data and have a known exponential $\chi^{2}(2)$ probability distribution. Peaks in the periodograms below a level of significance of $99.5 \%$ probability were rejected from the analysis. For a more detailed description of this CLEAN implementation cf. Kaufer et al. (1997).

Figure 5 shows the section of the two-dimensional power spectrum where the most significant periods are found in the photospheric lines, i.e., close to a period of $4.8 \mathrm{~h}$. Application of the iterative CLEAN algorithm as described above confirms that three significant periods with $P_{1}=4.810 \mathrm{~h}, P_{2}=4.672 \mathrm{~h}$, $P_{3}=4.967 \mathrm{~h}$ are present. The three periods are sorted by their associated power and significance with $P_{1}$ and $P_{2}$ being of comparable level but containing about twice as much power than $P_{3}$. The power within each detected period is not evenly distributed over the velocities but strongly peaked at zero velocity indicating stronger variability at the line center compared the wings of the line. Since the actually measured periods show some variations of the order of $1.25 \%$ as function of the velocity within the line profile, the periods given above have been measured from the position of the power peaks averaged over the full velocity interval $-v \sin i$ to $+v \sin i$, i.e., -265 to $+265 \mathrm{~km} \mathrm{~s}^{-1}$ (cf. Fig. 5, right plot).

To assure ourselves of the detected multi-periodicity of the photospheric variations we have to exclude that they are possibly caused by the complex window function of our observations. This is implicitly guaranteed by the CLEAN method, which we have used to identify the periods. However, we have verified the multi-periodicity in addition as part of the pulsation modeling of the variability, which will be described in Sect. 5.

The period analysis confirms the multi-periodicity of the photospheric line-profile variations in HD 64760 as had already been suspected from Fig. 2. It should be noted that the three periods are closely spaced in time with $\pm 3 \%$ separation and two periods show similar power, which are the prerequisites for the possible existence of beat periods on time scales much longer than the underlying periods themselves.

The two-dimensional velocity - phase diagrams of the photospheric Si III $\lambda 4553$ line constructed with the three identified periods are shown in Fig. 6 and clearly show the characteristic traveling features of three distinct prograde NRP modes. While the associated pulsation modes of $P_{1}$ and $P_{3}$ appear to be very similar, the pulsation mode of $P_{2}$ is clearly different as can be seem from the different slope of the NRP pattern.

Alternatively to the identification of the origin of the lineprofile variations (LPV) as non-radial pulsation patterns, similar LPVs could be produced by the rotational modulation by regular surface structures. For $P_{1}$ a crossing time of an individual surface feature from $-v \sin i$ to $+v \sin i$ is determined to be $13 \mathrm{~h}$ (according to a measured acceleration of $200 \mathrm{~km} \mathrm{~s}^{-1} / 4.81 \mathrm{~h}$ at the line center). This would corresponds to a stellar rotation period of $P_{\text {rot }}=26 \mathrm{~h}$. The breakup rotation velocity for HD 64760, however, is estimated to $P_{\text {rot, break }}=64 \mathrm{~h}$ assuming a stellar radius of $R=22 R_{\odot}$ and mass of $M=20 M_{\odot}$ (cf. KPS 2002). From this simple estimate it must be concluded that the observed LPVs cannot be explained by co-rotating surface structures. Further, the observation of closely spaced multiple periods is not compatible with rotational modulation where only the stellar rotation period or integer fractions of the rotation period can be observed.

Therefore, we have to conclude that the most likely source for the observed LPVs are multi-periodic non-radial pulsations. In Sect. 5 we will further substantiate this hypothesis by detailed modeling of the NRPs.

\subsection{The wind-sensitive $\mathrm{H} \alpha$ line}

The $\mathrm{H} \alpha$ line has been examined for periodicities in the same way as the photospheric lines using the iterative CLEAN algorithm described above. The power spectrum of $\mathrm{H} \alpha$ is very complex in particular in the frequency regime of $<1$ day $^{-1}$ where it is hardly possible to identify the most significant frequencies to which the CLEAN procedure shall be applied. Therefore, we had to closely inspect selected frequency ranges for which periodicities could be expected. First, we tried to verify the presence of the three photospheric pulsation periods close to $4.8 \mathrm{~h}$. 

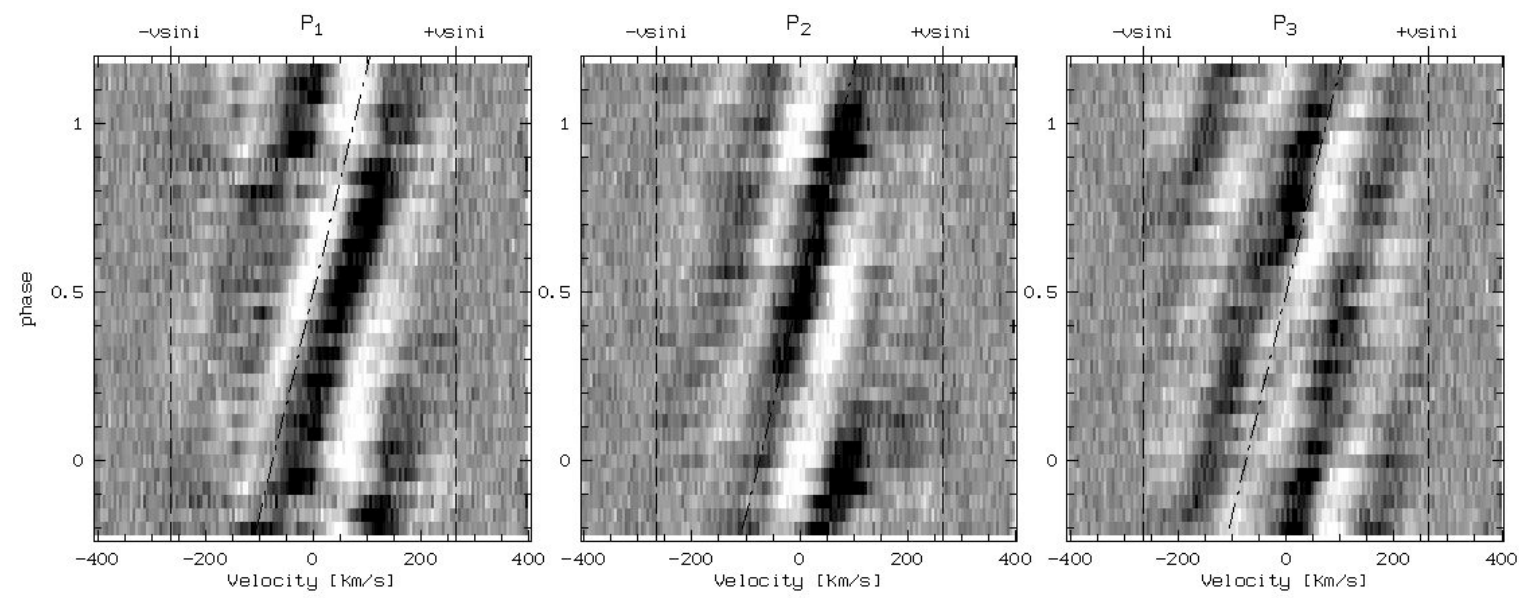

Fig. 6. Dynamical phase spectra of the Si III $\lambda 4553$ line. The observed spectra are phased with $P_{1}=4.810 \mathrm{~h}($ left $), P_{2}=4.672 \mathrm{~h}($ center $)$, $P_{3}=4.967 \mathrm{~h}($ right $) .25$ phase bins were used for the phase interval 0.0-1.0. All spectra are displayed with $\pm 0.3 \%$ cut levels. The measured typical acceleration of the features over the line center of $(\mathrm{d} v / \mathrm{d} \phi)=-175 \mathrm{~km} \mathrm{~s}^{-1} /$ cycle is indicated by a dot-dashed lines.
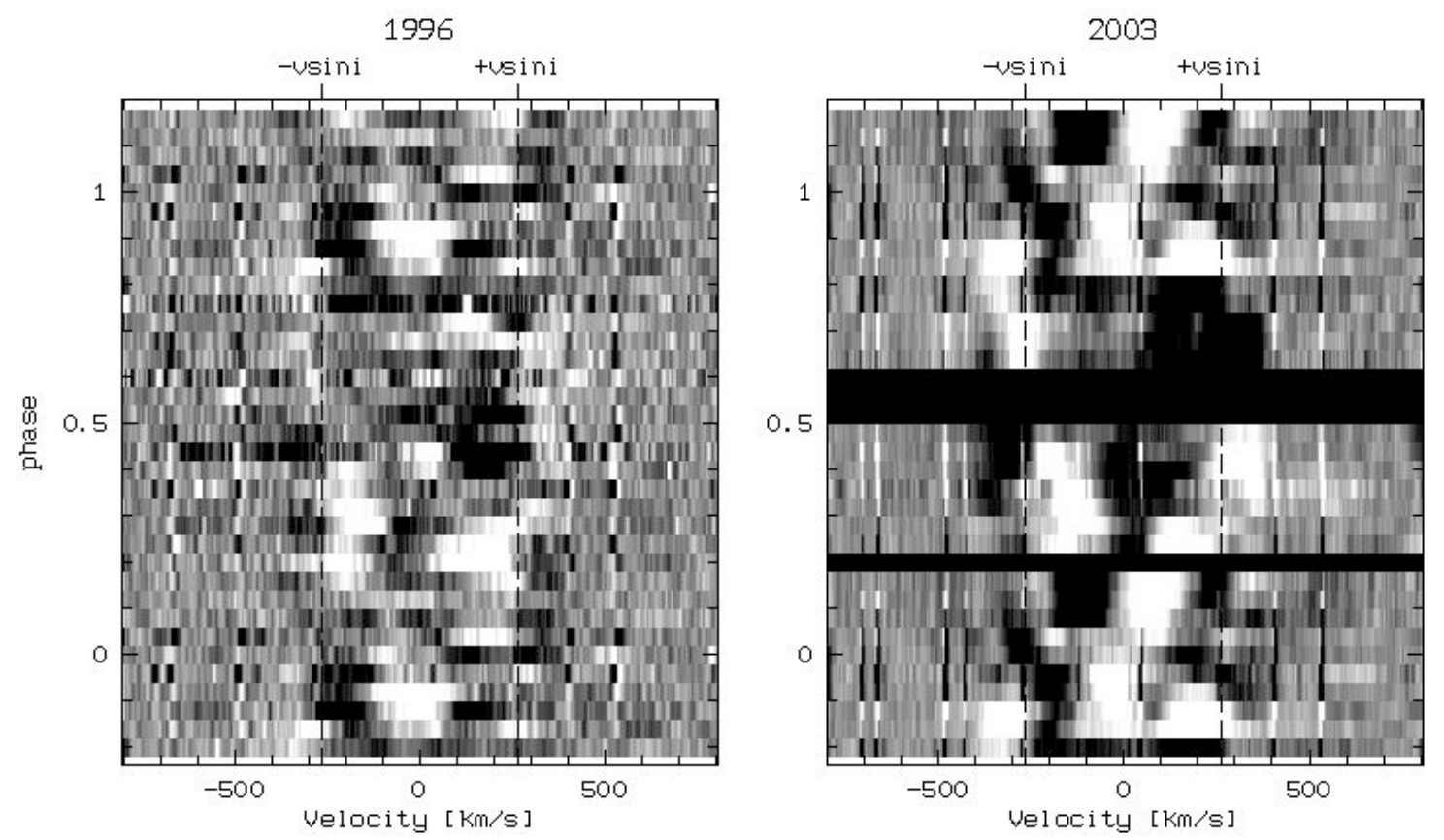

Fig. 7. Dynamical spectra of the $\mathrm{H} \alpha$ line phased with a period of 2.39097 days (cf. text). 25 phase bins were used for the phase interval $0.0-1.0$. Left 1996 HERos data set, right 2003 FEROS data set. Both dynamical spectra are displayed with $\pm 1 \%$ cut levels.

But no significant power could be found as expected from the lack of fast moving features in the dynamical spectrum of $\mathrm{H} \alpha$.

The short extent in time of the new FEROS data set (10 nights with several spectra within a few hours of each night) is not very well-suited to search for the continued presence of the 2.4-day wind modulation period as it was found in the IUE MEGA data set (15 days with continuous sampling) and confirmed in $\mathrm{H} \alpha$ with the HEROs data set (100 nights with 1 spectrum per night). Nevertheless, the CLEANed two-dimensional power spectra show significant power at a period of 2.4 days. If the $\mathrm{H} \alpha$ data sets of the HERos (1996) and the FEROs (2003) are both phased with the established period close to 2.4 days, a strikingly similar variability pattern is found for both optical data sets as can be seen in Fig. 7. Both data sets have been treated in exactly the same way by sorting the spectra in 25 phase bins (for phase 0.0-1.0) according to a period of 2.39097 days. Phase 0.0 is defined as the time of the observation of the first spectrum of the 2003 time series, i.e., MJD $=52685.0265$. The apparent accuracy of the period comes from the requirement to match the 1996 and the 2003 phase diagrams. However, this requirement is fulfilled by a set of discrete periods. Using phase-dispersion minimization of the combined $1996+2003$ data set, we have identified 20 such possible periods in the range from 2.37-2.41 days. Both time series have been resampled to a velocity resolution of $5 \mathrm{~km} \mathrm{~s}^{-1}$ and are displayed with $\pm 1 \%$ intensity cut levels. The respectively subtracted time-averaged $\mathrm{H} \alpha$ spectra from the 1996 data set (cf. KPS 2002, their Fig. 1) and the 2003 data set to compute the shown residuals are indistinguishable within the limitations of available $S / N$-ratio and achievable accuracy in the 
normalization of the stellar continuum. The variability pattern of the 2.4-day phased $\mathrm{H} \alpha$ line in Fig. 7 is very characteristic with " $\mathrm{X}$ " or " 8 "-shaped pattern for the pseudo-emission features with the "crossing point" close to phase 0.20 (or night 6 in the 2003 data set). KPS 2002 described the 2.4-day $\mathrm{H} \alpha$ variability in detail and tried to disentangle the different contributing components at distinct velocities in the line profile. All the features highlighted there can also be identified in the new FERos data set - however, the features appear to be more coherently traveling across the line profile. The resemblance of the two dynamical $\mathrm{H} \alpha$-spectra in Fig. 7 indicates that 2.4-day period has now been stable for at least eight years, i.e., the time span between the 1995 IUE, the 1996 HEROs and the 2003 FEROs campaigns. Such a long-term presence of a single modulation period requires a stable clock in the system. Stellar rotation has been favored by all previous works on HD 64760 , cf. Fullerton et al. (1997) and KPS 2002. In this work we will further investigate the possibility that stellar oscillations could act as stable clock in HD 64760, too. This possibility will be discussed in context with our pulsation and wind modeling in Sects. 5 and 6.

\section{NRP line-profile modeling}

The period analysis of the photospheric lines has revealed the presence of multi-periodic variability with three dominant periods. We have identified non-radial pulsations (NRPs) as the most likely origin of this variability and proceed now to confirm this hypothesis with detailed modeling of the LPVs.

In the following we first concentrate on the determination of the pulsation quantum numbers $l$ and $m$ of the NRP modes associated with the three pulsation periods. Only detailed modeling will allow us to test the consistency of the very complex observed LPVs with multi-periodic NRPs. However, because of this high complexity of the observations on one hand side and the very large parameter space on the other, most likely, an exact identification of the pulsation parameters is not possible with our observational data set.

We use the NRP modeling codes BRUCE and KYLIE (version 2.84) by Townsend (1997b) to examine the LPVs observed in the photospheric lines of HD 64760. BRUCE calculates the pulsational perturbations to the rotating stellar surface and includes pulsational velocity fields in all three spatial directions $(r, \phi, \theta)$, temperature fluctuations, variations in the visible surface area and the variations in the viewing angle of a given surface element. KYLIE derives in a second step the observable quantities from the perturbation fields provided by BRUCE by computing and integrating the observables like the observerdirected spectrum or flux of each surface element individually from an input grid of stationary atmosphere models.

The intrinsic line profiles and spectral energy distributions, which are required as input to KYLIE for the production of realistic non-radial pulsation LPVs and light curves, respectively, were computed on the basis of an extended grid of ATLAS 9 model atmospheres (Kurucz 1993) and subsequent BHT spectrum synthesis (Baschek et al. 1966) for solar abundances and a microturbulence of $\xi_{\text {micro }}=2 \mathrm{~km} \mathrm{~s}^{-1}$. The grid extends from 15000 to $31000 \mathrm{~K}$ in steps of $1000 \mathrm{~K}$ in effective temperature $T_{\text {eff }}$ and from 2.6 to 3.5 in steps of 0.1 in
Table 2. BRUCE/KYLIE model parameters.

\begin{tabular}{lrl}
\hline \hline & \multicolumn{2}{c}{ Model parameters } \\
\hline$T_{\text {pole }}$ & $29000 \mathrm{~K}$ & temperature at pole \\
$M$ & $20 M_{\odot}$ & total mass \\
$R_{\text {pole }}$ & $18 R_{\odot}$ & radius at pole \\
$v_{\text {equator }}$ & $265 \mathrm{~km} \mathrm{~s}^{-1}$ & rotation velocity at equator \\
$i$ & $90 \mathrm{deg}$ & inclination \\
\hline \multicolumn{3}{c}{ Derived parameters } \\
\hline$R_{\text {equator }}$ & $21.6 R_{\odot}$ & radius at equator \\
$\log g_{\text {pole }}$ & 3.23 & gravity at pole \\
$\log g_{\text {equator }}$ & 2.85 & gravity at equator \\
$T_{\text {equator }}$ & $23300 \mathrm{~K}$ & temperature at equator \\
\hline \multicolumn{3}{c}{ Observable parameters } \\
\hline$P_{\text {rot }}$ & $98.9 \mathrm{~h}(4.12 \mathrm{~d})$ & rotation period \\
$L_{\text {tot }}$ & $1.55 \times 10^{5} L_{\odot}$ & luminosity \\
$T_{\text {eff }}$ & $24600 \mathrm{~K}$ & effective temperature \\
\hline
\end{tabular}

gravity $\log g$. For the hotter atmospheres $(25000 \mathrm{~K}$ and higher), the ATLAS 9 models with the lowest $\log g$ values do not converge anymore. Fortunately, these grid points are not required in the KYLIE line-profile computations, since for a fast rotating star like HD 64760 the hottest regions are located at the stellar pole where the $\log g$ is high because of the rotational flattening of the star. The lower temperatures occur close to the equator, where also $\log g$ is found to be lower.

The stellar input parameters for BRUCE were chosen in such a way to reproduce the observable parameters of HD 64760 as given in KPS 2002 (their Table 1): a polar radius of $18 R_{\odot}$, a polar temperature of $29000 \mathrm{~K}$, a stellar mass of $20 \mathrm{M}_{\odot}$, an equatorial rotation velocity of $265 \mathrm{~km} \mathrm{~s}^{-1}$ ( $65 \%$ of the critical rotational velocity of $415 \mathrm{~km} \mathrm{~s}^{-1}$ ) and an inclination of $90^{\circ}$. These input values result in an equatorial radius of $21.6 R_{\odot}, \log g$ values of 3.23 and 2.85 at the pole and equator, respectively, an equatorial temperature of $23300 \mathrm{~K}$, and a rotation period of $98.9 \mathrm{~h}$ (4.12 days). The total luminosity and effective temperature, as seen from the observer, are $1.55 \times 10^{5} L_{\odot}$ and $24600 \mathrm{~K}$. The model parameters related to the stationary stellar model are summarized in Table 2.

\subsection{Pulsation mode parameters}

For the modeling of $n$ pulsation modes, BRUCE needs the pulsation periods $P_{n}$ as measured in the observer's system, the pulsation amplitudes $A_{n}$, the relative pulsation phases $\phi_{n}$ and the pulsation quantum numbers $m_{n}$ and $l_{n}$.

The pulsation quantum number $m$ is equal to the number of meridian circles on the stellar surface and can be estimated via the acceleration of the traveling features across the line center. From the phase diagrams in Fig. 6 we measure an acceleration of $(\mathrm{d} v / \mathrm{d} \phi)=-175 \pm 50 \mathrm{~km} \mathrm{~s}^{-1} /$ cycle. This translates with the purely geometrical relation $m_{n}(\mathrm{~d} v / \mathrm{d} \phi)_{n} / 2=2|v \sin i|$ into a possible range of values for $m$ of $-8.5<m<-4.7$.

While the $m$-value can be estimated with a reasonable accuracy by this method, the $l$-value remains ill-defined and can only be determined by detailed modeling of the LPVs. However, the presence of multiple pulsation periods and the 
Table 3. BRUCE/KYLIE model parameters.

\begin{tabular}{ccccccc}
\hline \hline \multicolumn{7}{c}{ Pulsation Modes } \\
\hline$n$ & $P_{n}[\mathrm{~h}]$ & $P_{n, \text { corot }}[\mathrm{h}]$ & $\phi_{n}[\mathrm{deg}]$ & $l_{n}$ & $m_{n}$ & $A_{n}\left[\mathrm{~km} \mathrm{~s}^{-1}\right]$ \\
\hline \multicolumn{7}{c}{ Model 1 } \\
\hline 1 & 4.810 & 7.872 & 180 & 8 & -8 & 5 \\
2 & 4.672 & 6.520 & 216 & 6 & -6 & 5 \\
3 & 4.967 & 8.302 & 97 & 8 & -8 & 3 \\
\hline \multicolumn{7}{c}{ Model 2 } \\
\hline 1 & 4.810 & 6.791 & 180 & 8 & -6 & 10 \\
2 & 4.672 & 6.520 & 216 & 6 & -6 & 5 \\
3 & 4.967 & 7.109 & 97 & 10 & -6 & 10 \\
\hline \multicolumn{7}{c}{ Model 3 } \\
\hline 1 & 4.810 & 7.872 & 180 & 6 & -6 & 5 \\
2 & 4.672 & 6.520 & 216 & 8 & -8 & 5 \\
3 & 4.967 & 8.302 & 97 & 6 & -6 & 3 \\
\hline
\end{tabular}

high but still finite $S / N$-ratio of the data will naturally limit the achievable accuracy of the $l$-value determination.

The amplitudes and relative phases of the pulsation modes have been chosen so that the best possible match to the observations is achieved: pulsation amplitudes of $3-10 \mathrm{~km} \mathrm{~s}^{-1}$ are needed to produce LPVs of the observed amplitudes of $\pm 1 \%$ in the Si III $\lambda 4553$ line; the relative phases have been adjusted to match the times of strongest variations in the observed time series, i.e., the times of constructive interference of the pulsation modes.

Our adopted pulsation mode parameters are summarized in Table 3. Two fundamental types of possible pulsation modes have been investigated, $l=-m$ (Models 1 and 3) and $l \neq-m$ (Model 2) and are discussed below.

The measured and derived stellar and pulsation-mode parameters in Tables 2 and 3 allow us to identify the expected pulsation-mode characteristics. The dimensionless pulsation frequency in the co-rotating frame $\omega_{\text {corot }}$ is defined as

$\omega_{\text {corot }}^{2}=\frac{\left(2 \pi / P_{\text {corot }}\right)^{2} R_{\text {pole }}^{3}}{G M}$

and reaches values of $\omega_{\text {corot }}^{2} \approx 30$ for the observed pulsation parameters of HD 64760. This places the three observed pulsation modes in the regime of the pressure- or $p$-mode pulsations (Townsend 1997a). This finding is consistent with the observed concentration of power at the line center in the twodimensional power spectrum (Fig. 5) indicating a strong preference of radial over horizontal velocity fields, which is the most direct characteristics of p-mode pulsations. In the NRP models, the ratio of the horizontal to the radial velocity amplitudes $k$ is described as $k=1 / \omega_{\text {corot }}^{2}$ and will provide a good representation of the power distribution over the line profiles (cf. Fig. 10 below).

\subsection{The case $I=-m$}

The analysis of the phase diagrams above provides a reasonable estimate of the $m$-value of each pulsation period but the degree $l$ remains ill-defined. $m$ can assume values from $-l$ to $+l$, i.e., the lowest acceptable value of $l$ in any case is $-m$. For a

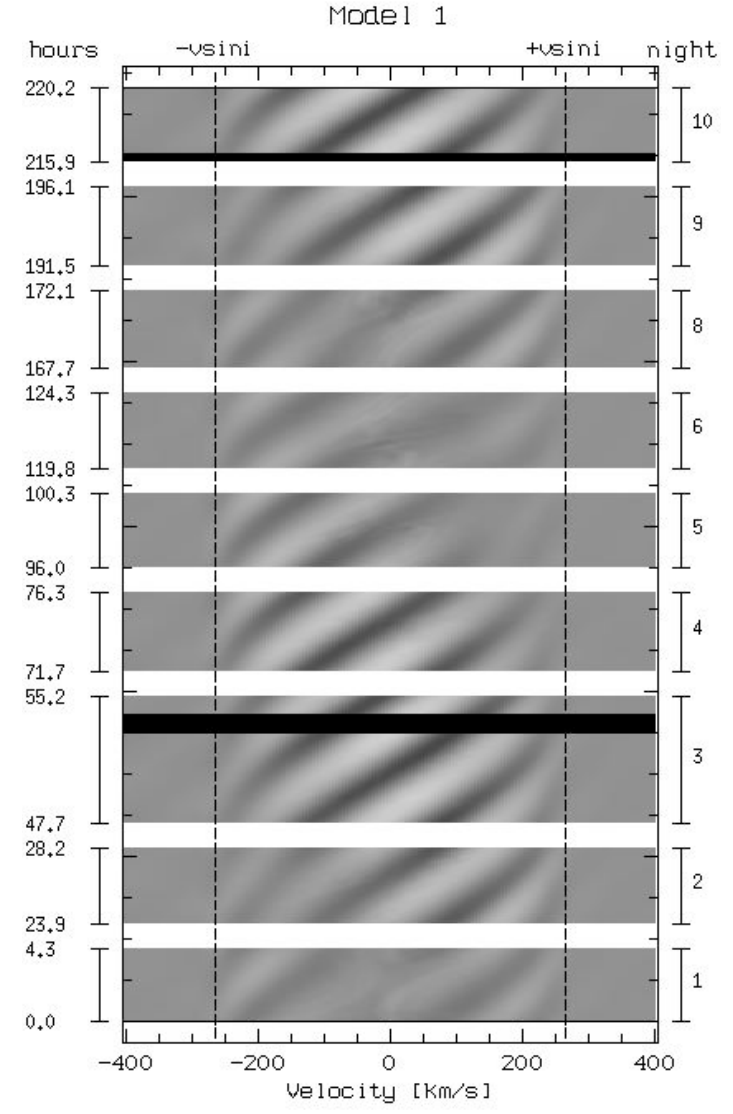

Fig. 8. Computed time series for the Si III $\lambda 4553$ line according to NRP Model 1 . Note the strong changes from night to night, which are due to the superposition of modes with different $m$ values. The choice of the $m$-values for the different periods leads in this model to a retrograde propagation of the beat pattern - consistent with the observations in Figs. 2 and 3. Displayed with $1 \%$ cut levels.

given choice of $l$, there will be $l-|m|$ nodes along any longitudinal line, and consequently, the stellar surface will be divided into $l-|m|+1$ sections in latitude. With an increasing number of zones on the stellar surface the mutual cancellation of the pulsation effects of neighboring zones will increase. Therefore, to produce the maximum observable effects, the smallest number of surface zones should be chosen, i.e., the case $l=-m$. This is the reason, why in many works on NRPs, this choice is preferred. However, usually the same amplitudes in the observables but with different characteristics can be achieved with higher amplitudes of the modulations in velocity or temperature in the case $l \neq m$.

We assume in our first test model (Model 1) $l=-m$ and $l=6$ for the $P_{2}=4.672 \mathrm{~h}$ and $l=8$ for the $P_{1}=4.810 \mathrm{~h}$ $P_{3}=4.968 \mathrm{~h}$ periods. For pulsations with different $m$-values, an interesting pattern for the resulting total pattern is produced: several temperature and velocity maxima emerge along the equator from the constructive or destructive interference of the pulsation modes. Depending on the period difference of the $m$ modes, this beat pattern moves across the stellar disk in the direction of the rotation (cf. our Model 3, Fig. 12), but also against this direction (this Model 1, Fig. 8). The relevant period is the beat period of the two modes. 

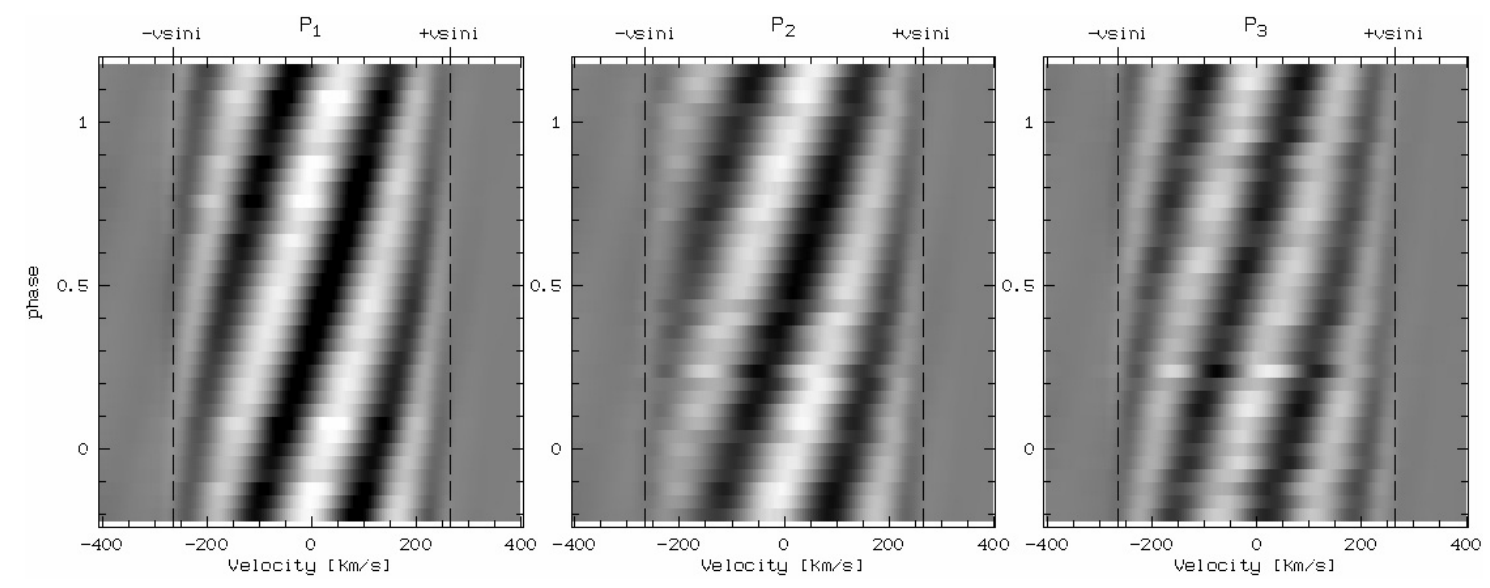

Fig. 9. Dynamical phase spectra for the the Si III $\lambda 4553$ line as computed for Model 1 . The spectra are phased with $P_{1}=4.810 \mathrm{~h}(l=8, m=-8)$, $P_{2}=4.672 \mathrm{~h}(l=6, m=-6), P_{3}=4.967 \mathrm{~h}(l=8, m=-8) .25$ phase bins were used for the phase range $0.0-1.0$. All spectra are displayed with $\pm 0.3 \%$ cut levels.

For a best possible comparison with the observations, we computed the NRP models with exactly the same sampling pattern as during the observations. Dynamical spectra of the computed time series of the Si III $\lambda 4553$ line and the photospheric part of the $\mathrm{H} \alpha$ line for Model 1 are shown in Figs. 8 and 11, respectively. The model for the Si III $\lambda 4553$ line reproduces the strong changes from night to night in the observed variability pattern. While the dominating non-radial pulsation features travel prograde over the line profile within a few hours, the constructive interference of the $P_{1}$ and $P_{2}$ pulsation modes travels retrograde over the line profile with a period determined by the beat period of the two dominant pulsation modes:

$\frac{1}{P_{\text {beat }}}=\frac{1}{P_{2}}-\frac{1}{P_{1}}=\frac{1}{4.672 \mathrm{~h}}-\frac{1}{4.810 \mathrm{~h}}=\frac{1}{162.8 \mathrm{~h}}$.

The maximum amplitude of the constructive interference is indeed observed on the nights 3 and 9 corresponding to the beat period of $162.8 \mathrm{~h}$ or 6.8 days. Further, the observed phases with low variability in nights 1 and 6 (cf. Figs. 2, 3) are quite well reproduced by this model with three beating periods. It is important to note here the directly observable secondary effects on the stellar wind as seen in the superimposed wind emission in the 3 line and - even more pronounced - in the $\mathrm{H} \alpha$ line. The emission feature travels across the line profile from red to blue and seems to follow the retrograde pattern and is strongest in the nights 2 to 4 and 6 to 9 .

Figures 9 and 10 show the phase diagrams and the twodimensional power spectrum as computed from Model 1 and have to be compared to the observations in Figs. 6 and 5, respectively. An excellent agreement with the observations is achieved with this multi-periodic NRP model, including the p-mode characteristics of the pulsation modes. On the other hand, Model 3, which produces a prograde traveling beat pattern (cf. Fig. 12) does not represent the observations very well and must be excluded.

The weakness of the predicted variations in the $\mathrm{H} \alpha$ line as shown in Fig. 11 (displayed with the same intensity cut levels as the Si III $\lambda 4553$ line in Fig. 8) is due to the intrinsically (Stark-) broadened photospheric $\mathrm{H} \alpha$-line profile, which tends to smear out the NRP-induced line-profile variations over the

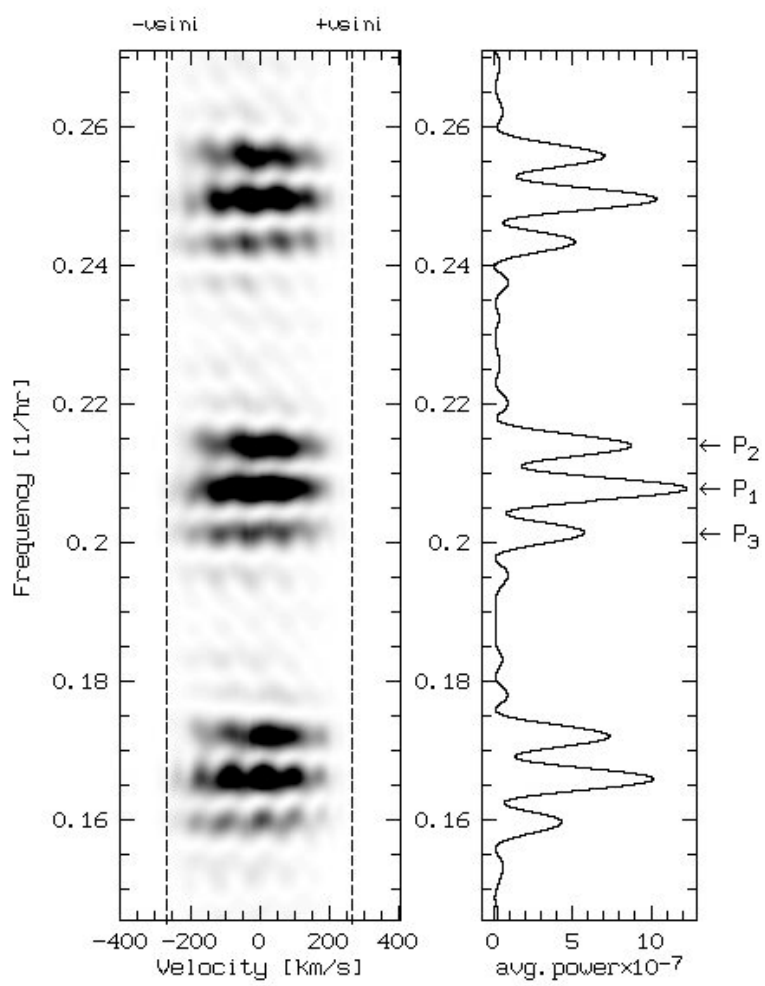

Fig. 10. Two-dimensional power spectrum of the SiIII $\lambda 4553$ line around a period of $4.8 \mathrm{~h}$ for Model 1 . The power distribution in velocity and frequency of the observations (Fig. 5) is reproduced very well by this model. The concentration of power at the line center is due to the p-mode characteristics of the pulsation modes.

extended line profile. This at first glance surprising result of the NRP modeling is consistent with the non-detection of any of the three NRP pulsation periods in the observed $\mathrm{H} \alpha$ time series.

On the other hand, the finding of NRP beat periods in the order of several days is of great importance for the investigation of the possible connections of (fast) photospheric variability on time scales of hours to (slow) wind variability on time scales of days. The possible implications will be discussed extensively 


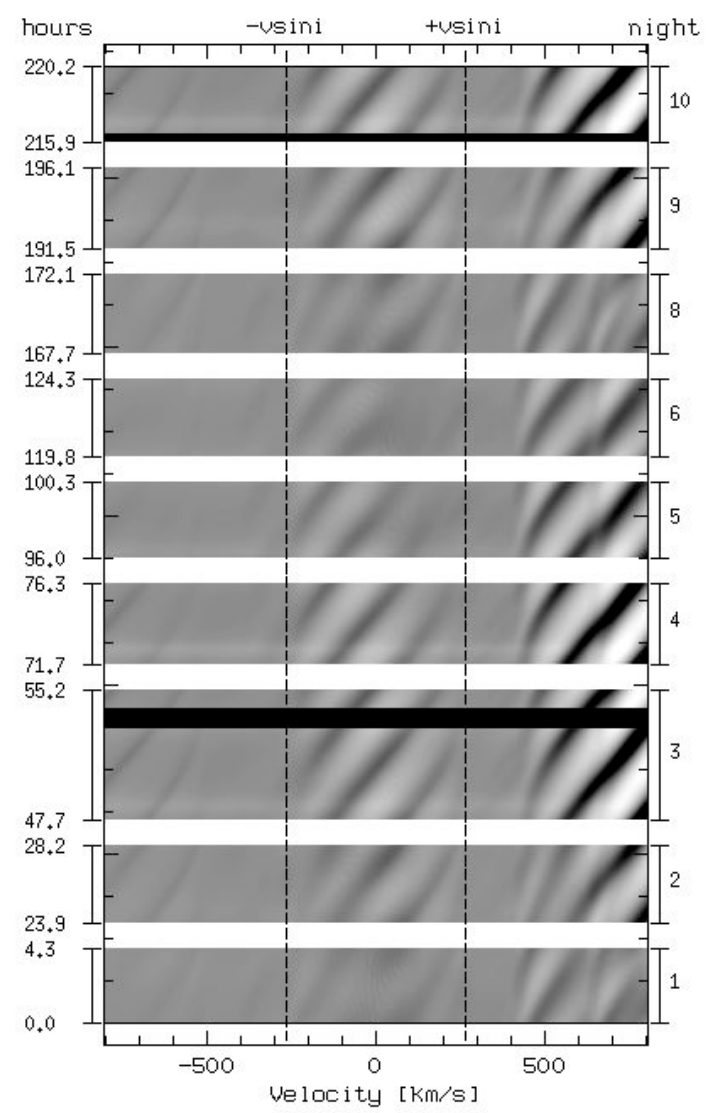

Fig. 11. Computed time series for the photospheric contribution of the $\mathrm{H} \alpha$ line according to Model 1. The expected variations in the line due to NRPs are very small consistent with the observations where the pulsation periods could not be detected. The strong variations at the right side are due to the neighboring C II $\lambda \lambda 6578,6582$ lines.

below in Sect. 6 where we will try to understand and model the effects of slowly varying photospheric surface structures on the stellar wind.

\subsection{The case $I \neq-m$}

The observed periods $P_{1}, P_{2}, P_{3}$ are closely spaced in time in the observer's reference frame. However, since the transformation of the periods to the co-rotating reference frame

$\frac{1}{P_{\text {corot }}(l, m)}=\frac{1}{P_{\text {obs }}(l, m)}+m \frac{1}{P_{\text {rot }}}$

only depends on the pulsation quantum number $m$, the pulsation periods are actually very different in the co-rotating frame for the case $l=-m$ as discussed above in Model 1. The observed close spacing of the observed periods would be in this case merely an unlikely coincidence.

Therefore, we tested with Model 2 the case of $l \neq-m$ but with $m$ identical for all three pulsation modes since in this case the close spacing in the observer's reference frame is naturally explained by a close spacing in the co-rotating reference frame.

In order to maintain the interesting beat patterns, which appear to be essential to explain the observed NRPs in the photospheric lines, we have to assume different $l$-values for the individual modes. In Fig. 13 we show the resulting line

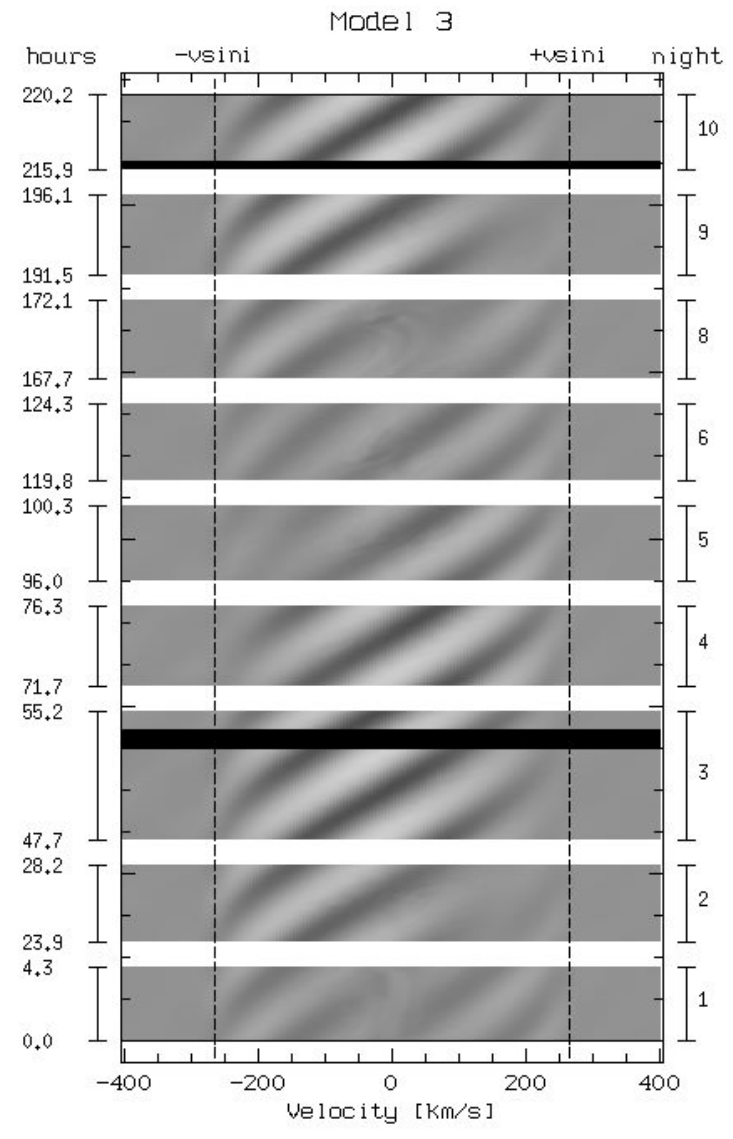

Fig. 12. Computed time series for the Si III $\lambda 4553$ line according to Model 3. The fact that the larger $m$-values has been assigned to the longer pulsation period leads to a prograde traveling beat pattern, which is not consistent to the observations. Displayed with $1 \%$ cut levels.

profile variations for one such case, with $l$-values of 10,8 and 6 and an $m$-value of -6 (Model 2 in Table 3). It can be seen that qualitatively similar changes can be produced as for the case of $l=-m$ and different $l, m$-values. With slightly larger velocity amplitudes of the pulsations the same amplitude of profile variations and the same power distribution over the line profile is produced. The increased velocity amplitudes are needed to compensate for the cancellation effects from surface zones of opposite phase. However, despite the fact that the two investigated cases with properly chosen model parameters lead to practically indistinguishable phase and power spectra, the actual resulting surface patterns are clearly more complex in the $l \neq-m$ case and in particular display strong differences between pole and equator within the same meridional sector of the star.

\subsection{Multi-line analysis}

One possibility to discriminate between $l=|m|$ and $l \neq|m|$ pulsation modes is a multi-line analysis of the NRPs. The strong variations of temperature and gravity from pole to equator in a fast rotating and pulsating star like HD 64760 provide additional diagnostics by the analysis of spectral lines with different temperature and gravity sensitivity. With polar/equator 
Model 2

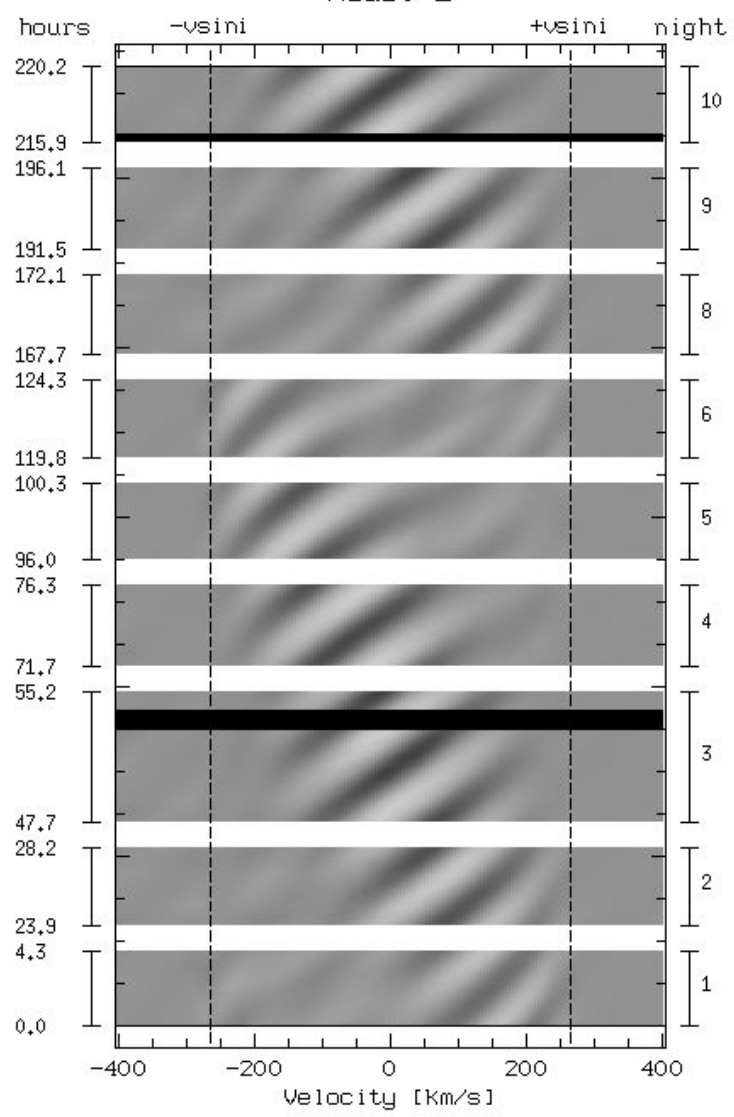

Fig. 13. Computed time series for the line Si III $\lambda 4553$ according to Model 2. Note the strong changes from night to night, which are due to the superposition of modes with different $l$ values. Displayed with $1 \%$ cut levels.

temperatures of $29000 / 23300 \mathrm{~K}$, the temperature sensitive He II $\lambda 4686$ line is of particular interest in this case.

Figure 14 shows the synthetic profiles for the the He II line and the neighboring $\mathrm{O}$ II $\lambda 4676$ line according to the parameters of our $l \neq|m|$ NRP Model 2. While the global appearance of the two lines is the same, distinct differences can be seen in the details of the NRP patterns, in particular in the smaller slope and the lesser extent in velocities of the features (i.e., they do not reach $\pm v \sin i$ ). These characteristics are as expected for the He II line, which is predominantly formed closer to the stellar pole at higher temperatures: (i) the number of features at a given time remains the same; (ii) $v \sin i$ becomes apparently smaller for that line since at the polar caps only smaller projected rotation velocities are seen; (iii) the slope of the traveling features has to become smaller since the pulsation periods have not changed.

The observed dynamical spectrum as shown in Fig. 15 corresponds well for the O II line as expected due to its similarities to the Si III line for which the model was constructed before and a good match with the observations was achieved. However, the model does not match for the He II line. In particular the amplitudes of the variations of the He II line are observed to be very small and hardly discernible with the given $\mathrm{S} / \mathrm{N}$ while in

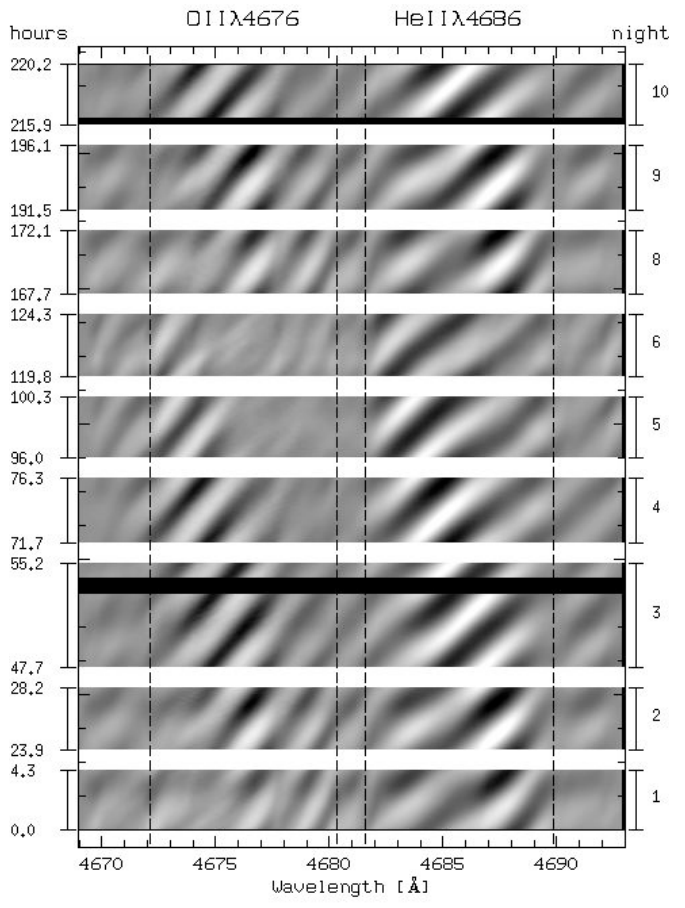

Fig. 14. Dynamical spectrum of the He II, O II spectral region computed according to the NRP Model 2 assuming adiabatic temperature effects (cf. observed data in Fig. 15). $\pm v \sin i$ for the O II (left) and the He II (right) lines are indicated.

the model the amplitudes are predicted to be even larger than for the O II line.

The fact that the models predict too large variations for the temperature sensitive He II line while less temperature sensitive lines are well represented indicates that the BRUCE models overestimate the pulsation-induced temperature variations. The models assume adiabatic temperature variations according to Buta \& Smith (1979). A good agreement between observation and model can be achieved if the temperature effects are reduced to $50 \%$ of the adiabatic value, which leads to a reduction of the amplitude of the variations by a factor of 5 . The corresponding dynamical spectrum is shown in Fig. 16. Interestingly, Buta \& Smith (1979) found already in their original work on the non-radial pulsator 53 Persei that the assumption of adiabaticity led to an overestimation of the pulsationinduced photometric variations by one magnitude. From our observations we have to conclude that the assumption of adiabaticity also breaks down for realistic pulsation models of HD 64760.

\subsection{Photometry}

Another possibility to discriminate between $l=|m|$ and $l \neq|m|$ pulsation modes is to compare the observed photometric variations with the photometric variations as predicted from NRP models.

Figure 17 shows the results from our BRUCE/KYLIE NRP simulations for the Johnson $\mathrm{V}$ filter for the two studied cases of NRP modes (i.e., Model 1 and 2 as defined in Table 3). For both cases, the maximum (peak-to-peak) amplitude of the variations 


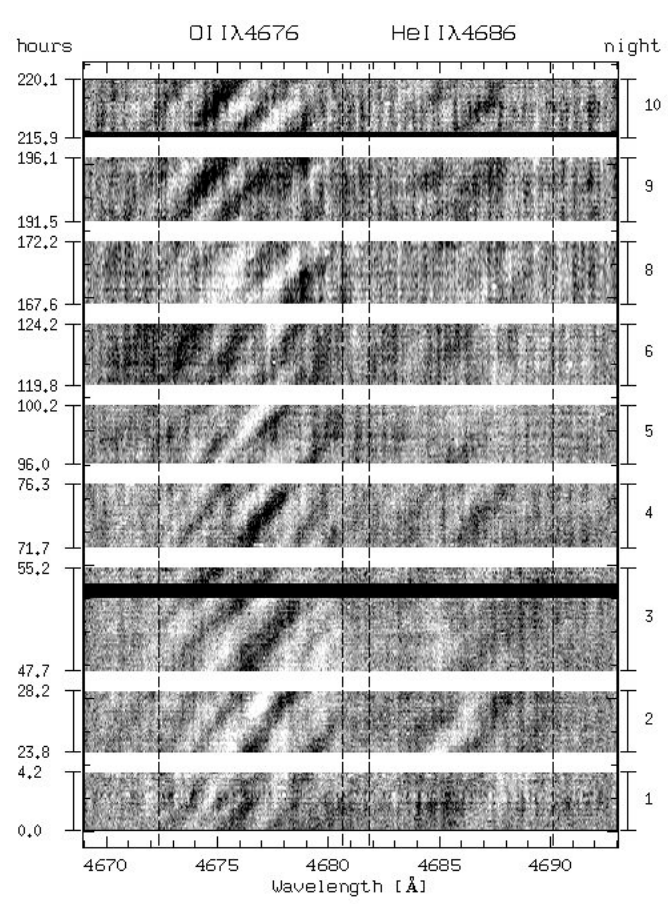

Fig. 15. Dynamical spectrum of the FEROs observations of the He II, O II spectral region. This dynamical spectrum has to be compared to the model computations as shown in Figs. 14 and 16. $\pm v \sin i$ for the O II (left) and the He II (right) lines are indicated.

is 20 mmag which is consistent with the photometric variations of up to $30 \mathrm{mmag}$ as frequently found in OB supergiants (Morel et al. 2004). the temperature effects in the NRP models are reduced to $50 \%$ of the adiabatic value as suggested by our analysis in Sect. 5.4, this leads to a reduction of the maximum amplitude of the photometric variations to $12 \mathrm{mmag}$.

While the high-frequency variations in the light curves are directly related to the dominant individual NRP modes with periods close to $4.8 \mathrm{~h}$, the overall evolution and the shape of the envelope of the light curves is governed by the beating of the pulsation modes. In particular the clearly different characteristics of the light curves at times of constructive $(t \approx 60,220 \mathrm{~h})$ and destructive interference $(t \approx 160 \mathrm{~h}$ ) is of high potential to discriminate between the two studied cases: while in the case of $l=|m|$ (Model 1) the amplitude of variations is minimal at times of constructive interference, the amplitude is maximal in the case of $l \neq|m|$ (Model 2) and vice versa at times of destructive interference. Further, while Model 1 predicts an increase of the average brightness by $10 \mathrm{mmag}$ at times of constructive interference, the variations of the average brightness according to Model 2 are reduced to some 5 mmag without clear relation to the evolution of the beat pattern.

HIPPARCos photometry (van Leeuwen et al. 1997) of HD 64760 (151 measurements over 550 days) displays dispersions of 7, 26, and $22 \mathrm{mmag}$ in the $H_{P}, B_{T}$ and $V_{T}$ passbands, respectively. The measured variations are comparable with our model predictions. However, no significant periodic signals can be found in the data sets. Unfortunately - to the authors' knowledge - no other suited photometric data sets are available for HD 64760 to verify the predictions of the NRP models.

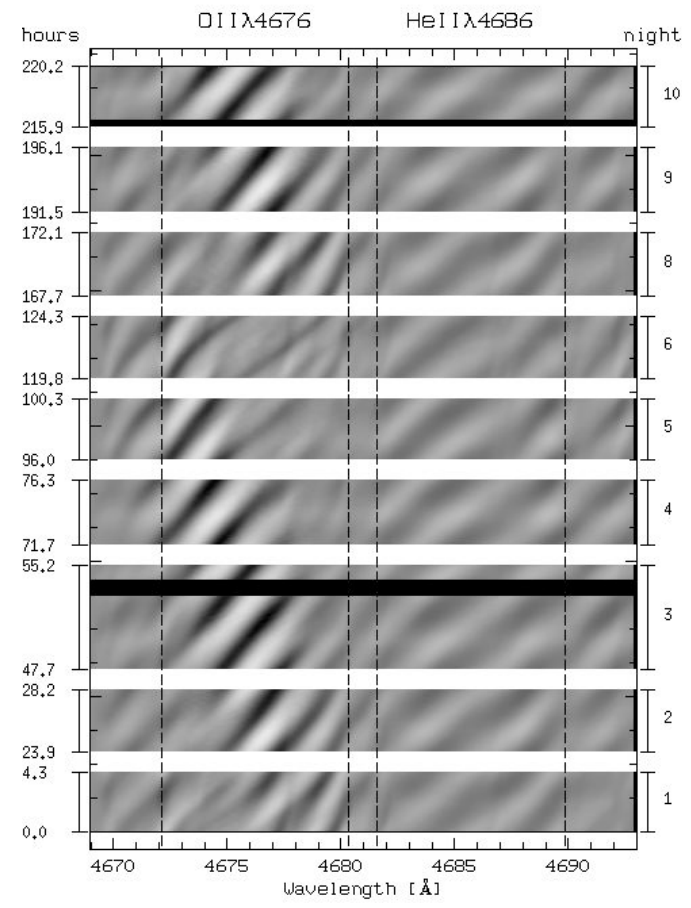

Fig. 16. Dynamical spectrum of the He II, O II spectral region as computed from Model 2 but with the pulsation-induced temperature effects reduced to $50 \%$ of the adiabatic value (cf. observed data in Fig. 15). $\pm v \sin i$ for the O II (left) and the He II (right) lines are indicated.

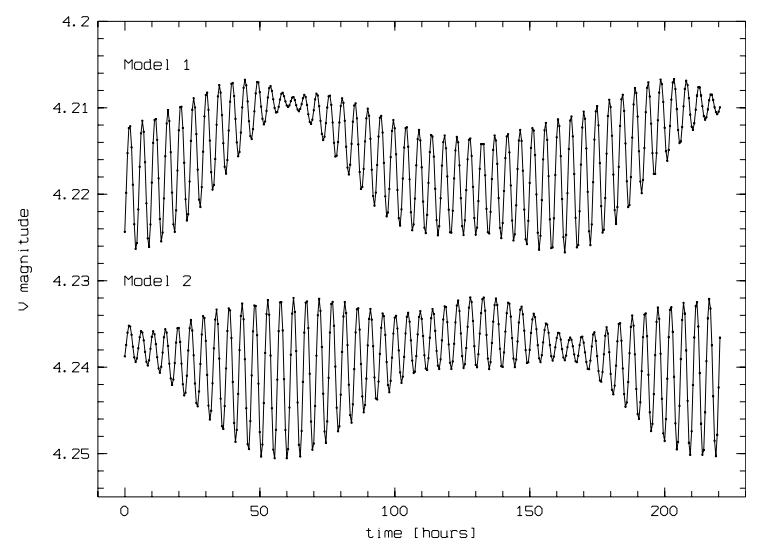

Fig. 17. Photometric variations in the Johnson $V$ band as predicted by NRP Model 1 (upper light curve) and NRP Model 2 (bottom light curve) for the full 10-day observing run and with a time sampling of $30 \mathrm{~min}$. The magnitude scale of the model has been adjusted to match the $V$ magnitude of HD 64760 from the literature $(V=4.24$, Hoffleit \& Jaschek 1982). The synthetic light curve for Model 1 has been shifted by $-0.025 \mathrm{mag}$ for better display.

The required photometric accuracy of a few mmags appears technically feasible for a dedicated photometric monitoring campaign, which could put additional rigid constraints on the NRP modeling, in particular on the selection of the pulsation mode parameters.

\section{Stellar-wind modeling}

One of the key questions for understanding the nature and origin of the wind variability in hot stars is its connection to the 
stellar photosphere. It is widely assumed, that the winds are modulated by a mechanism related to the photospheric rotation, presumably patches on the stellar surfaces produced either by non-radial pulsation (NRP) patterns or magnetic surface structures ("spots"). The spatial structures in the photospheres locally change the lower boundary condition of the stellar wind, which in turn causes localized structural changes in the wind. These structural changes modulate the observed stellar wind profiles as they are dragged through the line of sight to a distant observer by underlying rotation of the star.

In KPS 2002 we have introduced a simple 1.5D-model (dubbed ROTBALMER) for a differentially rotating stellar wind to model the double emission $\mathrm{H} \alpha$ profile of HD 64760. The model is based on the work and description of Petrenz \& Puls (1996) and allows a correct handling of the rotationally twisted resonance zones in the line formation of wind lines like $\mathrm{H} \alpha$. The model makes the simplified assumption of a spherically symmetric density stratification throughout the wind.

To estimate the effects of a geometrically structured rotating wind, we have introduced in the wind model density enhancements with respect to the local ambient wind following a streak-line geometry as described by Fullerton et al. (1997). The streak line is characterized by the density contrast $\rho_{\mathrm{c}}$ with respect to the ambient wind, the angular extension $\phi_{\mathrm{s}}$ on the stellar surface and the rotation velocity $v_{\mathrm{s}}$ of the foot point. Usually, $v_{\mathrm{S}}$ is set equal to the rotation velocity $v_{\text {rot }}$ of the star corresponding to a streak line emerging from the stellar surface and therefore locked to the stellar rotation.

\subsection{The case $v_{s}=v_{\text {rot }}$}

The most natural clock for a strictly periodic wind modulation is provided by the stellar rotation. For HD 64760 the upper limit for the stellar rotation period of 4.2 days is within the expected errors close to twice the 2.4-day modulation period. If this estimate is correct, the modulating structure on the stellar surface must be also persistent over such a time scale and geometrically divide the stellar surface in two structures with 180 degree azimuthal separation.

Our first RoTBALMER wind test model (Model 1) tries to simulate this situation with the presence of $n_{\mathrm{s}}=2$ diametrically opposed streak lines in the equatorial plane and with their foot points rotating at the same speed as the stellar surface, i.e., $v_{\mathrm{s}}=v_{\text {rot }}:=v_{\text {equator }} \sin i$. The complete set of ROTBALMER model parameters used in the model is given in Table 4 and is basically identical to the parameter set used in KPS 2002 except for the additional parameters to describe the streak lines. The geometry of the emerging streak lines is shown in Fig. 18.

Figure 19 shows the dynamical $\mathrm{H} \alpha$ spectrum as produced by this model with two co-rotating streak lines. We have again computed the model spectra with the same timings as the observations to ease their comparison. If the model is compared to the observations in Fig. 4 it is found that neither the velocity evolution of the pseudo-emission and absorption features (the " $X$ " or " 8 " shaped pattern) is well represented nor the time scales of the variations. The dominant time scale is due to the presence of two streak lines equal to half the stellar rotation
Table 4. ROTBALMER model parameters.

\begin{tabular}{|c|c|c|}
\hline \multicolumn{3}{|c|}{ Model parameters } \\
\hline$T_{\text {eff }}$ & $24600 \mathrm{~K}$ & effective temperature \\
\hline$R_{\text {equator }}$ & $21.6 R_{\odot}$ & radius at equator \\
\hline$v_{\text {equator }}$ & $265 \mathrm{~km} \mathrm{~s}^{-1}$ & velocity at equator \\
\hline$i$ & $90 \mathrm{deg}$ & inclination \\
\hline$v_{\text {inf }}$ & $1500 \mathrm{~km} \mathrm{~s}^{-1}$ & wind terminal velocity \\
\hline$v_{\min }$ & $10 \mathrm{~km} \mathrm{~s}^{-1}$ & wind start velocity \\
\hline$\beta$ & 0.8 & wind acceleration parameter \\
\hline$\dot{M}$ & $9 \times 10^{-7} M_{\odot} / \mathrm{yr}$ & mass-loss rate \\
\hline $\mathrm{Y}_{\mathrm{He}}$ & 0.10 & helium abundance $n(H e) / n(H)$ \\
\hline $\mathrm{I}_{\mathrm{He}}$ & 2 & free electrons per Helium atom \\
\hline \multicolumn{3}{|c|}{ Streak-line parameters } \\
\hline \multicolumn{3}{|c|}{ Model 1} \\
\hline$n_{\mathrm{s}}$ & 2 & Number of streak lines \\
\hline$\rho_{\mathrm{c}}$ & 1.3 & Density contrast w.r.t. ambient \\
\hline$\phi_{\mathrm{s}}$ & $50 \mathrm{deg}$ & Angular extension $(2 \arctan 0.5)$ \\
\hline$v_{\mathrm{s}}$ & $265 \mathrm{~km} \mathrm{~s}^{-1}$ & Foot-point rotation velocity \\
\hline \multicolumn{3}{|c|}{ Model 2} \\
\hline$n_{\mathrm{s}}$ & 2 & Number of streak lines \\
\hline$\rho_{\mathrm{c}}$ & 1.3 & Density contrast w.r.t. ambient \\
\hline$\phi_{\mathrm{s}}$ & $50 \mathrm{deg}$ & Angular extension $(2 \arctan 0.5)$ \\
\hline$v_{\mathrm{s}}$ & $-80.5 \mathrm{~km} \mathrm{~s}^{-1}$ & Foot-point rotation velocity \\
\hline
\end{tabular}

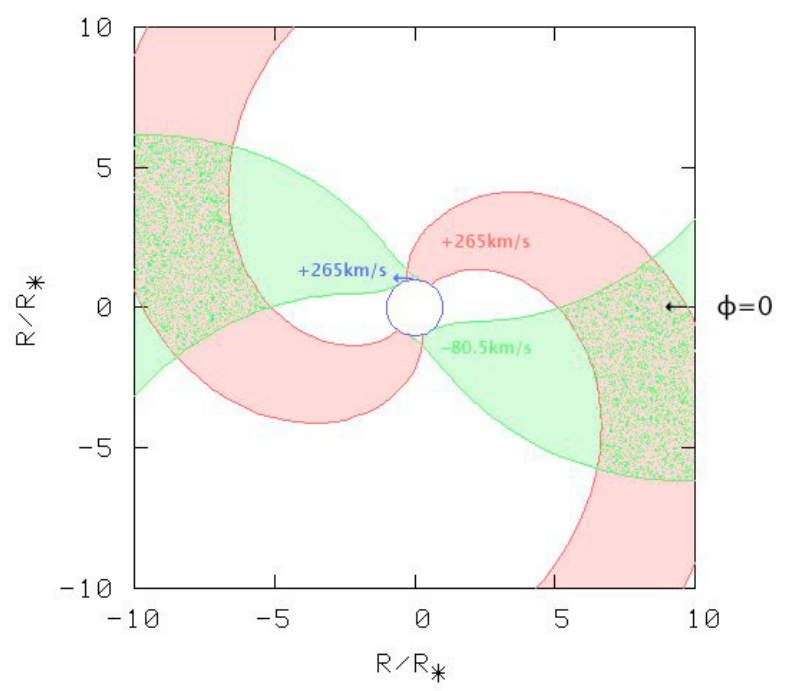

Fig. 18. Streak-line geometry for $v_{\text {rot }}=+265 \mathrm{~km} \mathrm{~s}^{-1}$ and $v_{\mathrm{s}}=$ $+265 \mathrm{~km} \mathrm{~s}^{-1}$ (Model 1) and $v_{\text {rot }}=+265 \mathrm{~km} \mathrm{~s}^{-1}$ and $v_{\mathrm{s}}=-80.5 \mathrm{~km} \mathrm{~s}^{-1}$ (Model 2). The observer looks at the star from the right at model phase $\phi=0$.

period, i.e., $4.2 / 2=2.1$ days - the model had been designed to exactly match this condition. The model further predicts $\mathrm{H} \alpha$ line-profile variations with blue- and redwards accelerating features as expected for two symmetric streak lines rotating through the line of sight of the observer. The features are seen from zero velocity up to the terminal velocities of the wind. This result is comparable to the $\zeta$ Puppis (O4 I(n)f) models by Harries (2000) who investigated the effects on the $\mathrm{H} \alpha$ line profile of a narrow and high-contrast one-armed spiral in the rotating wind of this prototypical O-type star. Indeed, we have used the model by Harries (2000) to verify the functionality of our 


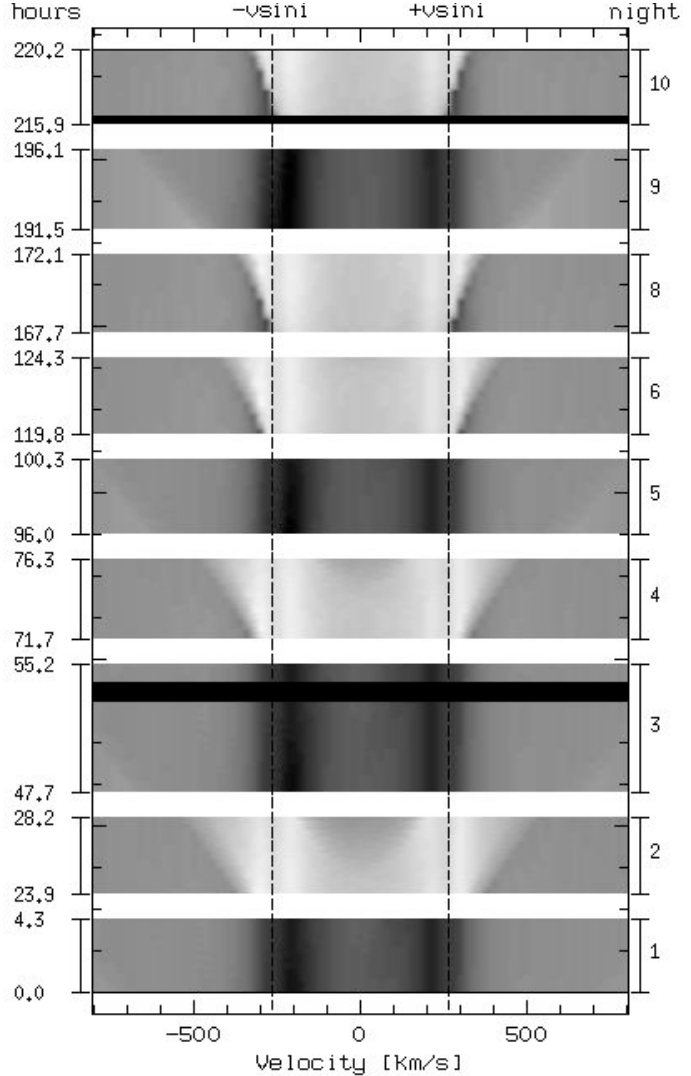

Fig. 19. $\mathrm{H} \alpha$ Rotbalmer Model 1 with $v_{\mathrm{s}}=v_{\text {rot }}=265 \mathrm{~km} \mathrm{~s}^{-1}$.

simpler ROTBALMER code and can report that we find a good agreement in the modeled LPVs.

\subsection{The case $v_{s} \neq v_{\text {rot }}$}

Our second $\mathrm{H} \alpha$ rotating structured wind model assumes again streak lines spaced at equal angles along the equator. However, in contrast to the previous case, we do not assume that the foot points of the streak lines are fixed on the star and follow its rotation. In contrast, we assume that the streak lines follow the pulsation pattern, as resulting from the superposition of the three detected NRP modes. This pattern does not follow the rotation, but has a pattern speed slower than the rotation or - as in our case - can even move (in the observer's reference frame) in the opposite direction as the rotation. While this does not change the stream line of an individual particle, the streak lines can change significantly (cf. below). The number of streak lines depends on the pulsation pattern. In the case of $l=-m$, two interfering pattern with different $m$ produce $m_{1}-m_{2}$ maxima along the equator. If $l \neq-m$ and $m$ equal for both modes, $m$ maxima along the equator appear. For arbitrary values of $l$ and $m$ (or more than two interfering modes), the interference pattern can become very complex. Therefore, we investigate here the case $l=-m$ (corresponding to our NRP Model 1), which is more attractive as lower boundary condition for a wind model with equatorial streak lines because it favors larger pulsation (beat-) amplitudes at the stellar equator. This second $\mathrm{H} \alpha$ rotating structured wind model assumes again $n_{\mathrm{s}}=2$ streak lines diametrically opposite at the equator corresponding to the 2 maxima in the beat amplitudes as created for $m_{1}-m_{2}=-2$.

The period in which the beat pattern moves across the star is related to the beat period. Therefore, periods significantly longer than the individual pulsation periods can result. The beat period is not related to the rotation period of the star, but only to the pulsation periods. If we consider the two strongest pulsation modes $P_{1}$ and $P_{2}$ only, we obtain $P_{\text {beat }}=162.8 \mathrm{~h}$ (cf. Eq. (2)). The apparent rotation velocity of the maxima of the beat pattern is then computed as:

$$
\begin{aligned}
v_{\mathrm{s}} & =v_{\text {rot }}\left(m_{1}-m_{2}\right) \frac{P_{\text {rot }}}{P_{\text {beat }}} \\
& =265 \mathrm{~km} \mathrm{~s}^{-1}(-8--6) \frac{98.9 \mathrm{~h}}{162.8 \mathrm{~h}} \\
& =-80.5 \mathrm{~km} \mathrm{~s}^{-1} .
\end{aligned}
$$

The geometry of the streak line changes considerably compared to the co-rotating case as is illustrated in Fig. 18. The stream line of the individual particle ejected from the stellar surface does not change and is ruled by the $\beta$-law acceleration with the ambient wind in radial and by the conservation of angular momentum in azimuthal direction. However, since the spot from where the particles are ejected either rotates fixed relative to the stellar surface $\left(v_{\text {rot }}=v_{\mathrm{s}}=+265 \mathrm{~km} \mathrm{~s}^{-1}\right)$ (Model 1) or moves in the opposite direction of the rotation $\left(v_{\text {rot }}=+265 \mathrm{~km} \mathrm{~s}^{-1}\right.$ and $v_{\mathrm{s}}=-80.5 \mathrm{~km} \mathrm{~s}^{-1}$ ) (Model 2) the resulting streak line, i.e., the snapshot of all ejected particles for a given time, appears substantially different (cf. Fig. 18).

Figure 20 shows the correspondingly computed ROTBALMER model (Model 2). Despite being far from perfect, this model with a counter-rotating pulsation beat pattern and two streak lines emerging from the maxima of beat amplitudes does show some resemblance with the observed " 8 " or " $X$ "-shaped pattern in the dynamical spectra in Fig. 4. Particularly remarkable is the evolution of the pseudo-emission features from wind velocities beyond $\pm v \sin i$ to zero-velocity in nights 1 to 4 and the phase of no variability in night 5, which are mostly responsible for the resemblance with the observed "crossing" features. Also the lack of features accelerating with the wind matches the observations better than the first model. Most important, also the time scales based on the beat period and the presence of two maxima in the beat amplitudes on the stellar equator match the observations surprisingly well. Clearly, the observed pseudo-emission and absorption features are more "discrete" in velocity and time than the model prediction. But considering the large simplifications and the complete lack of physics in the transition zone from the pulsating photosphere to the base of the wind in our model, the result is encouraging for further detailed modeling.

The important finding from our simplistic model therefore is, that the constructive interference of multi-periodic NRPs could play the crucial rule of providing the surface structure moving with the right time scales at the base of the wind to match (i) the observed base-of-the-wind variability $(\mathrm{H} \alpha)$ and (ii) the outer-wind structures rotating through the observer's line of sight as observed in the UV. 


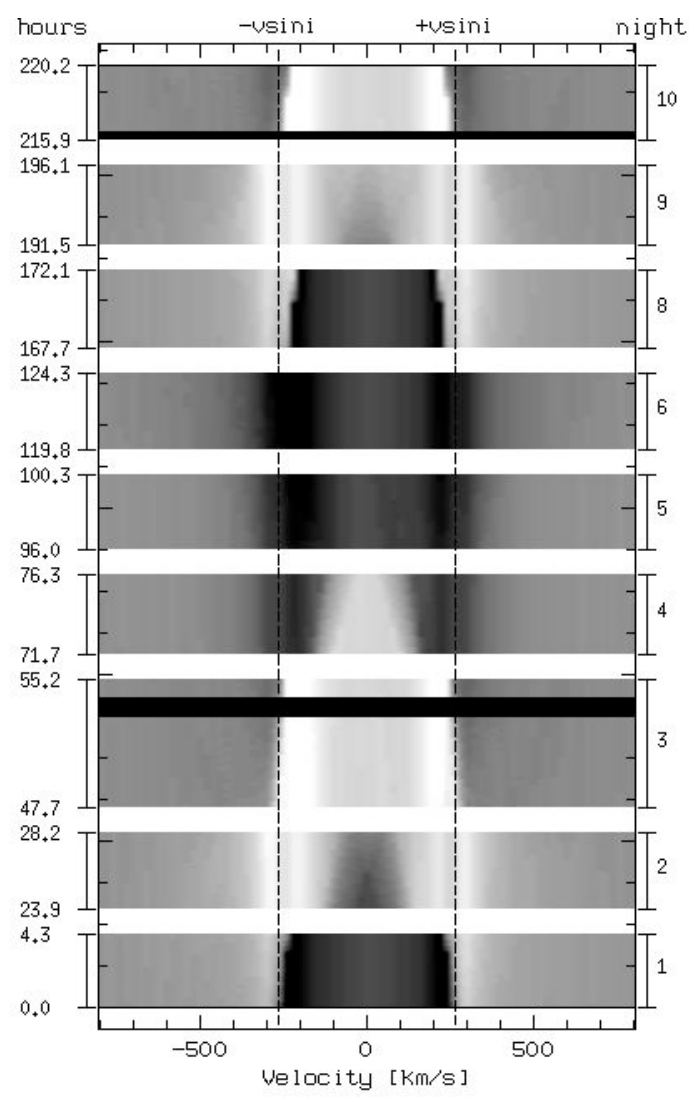

Fig. 20. $\mathrm{H} \alpha$ Rotbalmer Model 2 with $v_{\mathrm{s}}=-80.5 \mathrm{~km} \mathrm{~s}^{-1}$ corresponding to a beat period of $162.8 \mathrm{~h}$.

\section{Discussion}

The new high-resolution optical times series presented in this work allowed us to further investigate the line-profile variability of the B-supergiant HD 64760 in the photosphere and at the base of the wind. This new FEROS data set provides detailed information to extend the HEROs data set (KPS 2002).

The high quality and the good sampling of the photospheric and wind variability over more than 2 rotational cycles of the star allowed us to reveal the presence of multi-periodic lineprofile variations (LPVs) in the stellar photosphere. The three closely spaced periods could be identified as prograde highorder non-radial pulsation (NRP) modes with $l$ and $|m|$ values in the range $6-8$. The first detection of high-order NRPs at a period of 0.1 days had been reported by Baade (1984) however, his data set was not adequate for a possible detection of closely spaced multi-periodic NRPs. Nevertheless, even the quality of our data set did not allow a very precise determination of the three pulsation modes from the small-amplitude $(<0.5 \%)$ LPVs. Therefore, we tested the two fundamental cases $l=-m$ and $l \neq-m$ via detailed modeling of the multiperiodic LPVs. Both cases allow us to represent the observations equally well. However, we favor the case $l \neq-m$ to account for the fact that the observed $3 \%$ splitting into the three periods $\left(P_{1}=4.810 \mathrm{~h}, P_{2}=4.672 \mathrm{~h}, P_{3}=4.967 \mathrm{~h}\right)$ is a true physical and asteroseismologically significant period splitting in the star's co-rotating frame, which implies that the $m$-values of the three modes have to be equal and different from $l$-values (our Model 2, cf. Table 3). In the case of rotational splitting of pulsation modes, the expected magnitude of the effect is such that the beating of rotationally split frequencies is of the order of the rotational frequency. Considering the measured split frequencies, it is found that their beat frequency is roughly consistent with the estimated rotational frequency of HD 64760. However, additional complications to this simple estimate have to be considered for an asteroseismological application of measured rotational splitting (Kawaler et al. 1999). It should be kept in mind that - if our preference for the $l \neq-m$ case is correct - we are facing highly complex NRP surface patterns in HD 64760. For this reason we were restricted to use the geometrically simpler $l=-m$ model for our subsequent modeling of stellar wind structure rooted in photospheric NRPs.

The shortness of the measured periods and the absence of significant horizontal velocity fields clearly identifies the pulsation modes as p-modes. The pulsation parameters with periods of a few hours as found for HD 64760 compare well with the findings for other O-type stars (cf. e.g. Henrichs 1999 for a compilation). It is particularly interesting that the first NRPs in early-type supergiants were discovered in $\gamma$ Arae (B1 ib) by Baade \& Ferlet (1984) with $\gamma$ Arae being almost a twin to HD 64760 with respect to the stellar parameters, including the very high value of $v \sin i$. In particular the short-term variability in the photospheric lines of $\gamma$ Arae with a derived period of 0.17 days and $l=10$ is very reminiscent of the variability seen in this work in HD 64760. However, it is important to note that the variability in the UV wind-lines of $\gamma$ Arae is quite distinct from the periodic behavior of HD 64760, cf. Prinja et al. (2002).

Fullerton et al. (1997) had proposed as one possible source of regular surface structure in HD 64760 NRP patterns, which are locked to the 4.8-day rotation of the star, i.e., low-order g-modes with observed pulsation periods of 2.4 and 1.2 days and $l=-m$-values of 2 and 4 , respectively. However, this scenario would imply an infinitely long pulsation period in the corotating frame (cf. Eq. (3)), which appears not to be very physical. Further, we have no evidence from models of pulsational instabilities for very long pulsation periods, for which the rotation could become the dominant time scale in the observer's frame.

KPS 2002 attributed the observed 2.4-day period in the $\mathrm{H} \alpha$ variability to modulations of the inner flanks of the emission humps at photospheric velocities to complex width variations of the underlying photospheric $\mathrm{H} \alpha$ profile and tried to connect these variations directly to the effect of NRP-induced LPVs of the photospheric $\mathrm{H} \alpha$ profile. The fact that the strongest variability in the line profile is seen close to velocities of $\pm v \sin i$ was taken as hint for the presence of strong horizontal velocity fields, i.e., the presence of low-order g-mode pulsations. The marginal detection of NRP-like LPVs in the He I $\lambda 4026$ line further supported this hypothesis. With the new 2003 data set and the modeling results as presented in this work, this picture has to be refined. As shown in Sect. 5, NRPs are not expected to appear directly as measurable LPVs of the photospheric $\mathrm{H} \alpha$ profile due to smearing of the variations over the line profile as caused by the Stark-broadening of the intrinsic line profiles. Further, the new data set could not confirm the 
existence of NRP-induced LPVs on time scales of the wind modulation period but revealed the presence of multi-periodic NRPs, which are identified as p-mode pulsations with small horizontal velocity fields and periods of hours. Therefore, the observed $\mathrm{H} \alpha$ wind variations on time scales of days can only be attributed to secondary effects possibly linked to the presence of NRPs as discussed below. The interpretation of the observed $\mathrm{H} \alpha$ wind variations is further complicated by the presence of the differentially rotating wind: our simulations with the ROTBALMER code indicate that a simple direct mapping of velocities in the line profile to a location in the stellar wind is not possible in the presence of rotationally twisted resonance zones. Indeed, any large-scale wind variability (like rotating streak lines) is primarily mapped into LPVs close to $\pm v \sin i$. The power distribution over the line profile as e.g. derived from the models shown in Figs. 19 and 20 display four power peaks blue- and red-wards of $\pm v \sin i$ which nicely reproduces the observed power distribution of the observed $\mathrm{H} \alpha$ variability (cf. KPS 2002, their Fig. 3, blue and red peaks "A" and "D").

The most striking consequence from our finding of multimode NRPs with periods of a few hours is the presence of beat periods of several days. These beat periods can be directly seen in the observed and modeled photospheric LPVs as retrograde patterns traveling slowly (days) over the line profile superimposed on the fast (hours) prograde patterns of the individual pulsation modes (see e.g. Fig. 8). Simultaneously, we find the variability of the mostly wind-sensitive $\mathrm{H} \alpha$ line to be of the same time scales. Even some characteristics of the $\mathrm{H} \alpha$ line-profile variability like phases of low wind variability seem to coincide with phases of small beat amplitudes in the photospheric NRPs (cf. Fig. 4). The He I 26678 line (cf. Fig. 3), which shows on top of the photospheric NRP patterns some wind emission features, demonstrates directly the connection of the retrograde traveling NRP beat pattern with the wind emission, which apparently follows the beat pattern over the line profile (notably over the days 6-9). On the other hand, the NRP models of $\mathrm{H} \alpha$ have shown us that the photospheric NRPs themselves do not contribute to the LPVs of $\mathrm{H} \alpha$ (cf. Fig. 11).

This is obviously an exciting finding since it provides us for first time with direct observational evidence for a connection of photospheric multi-periodic NRPs with spatially structured winds. The constructive or destructive interference of multiple photospheric pulsation modes with time scales of a few hours (the intrinsic stellar pulsation time scale of HD 64760 is $<1$ day if a pulsation constant of $Q=0.04$ days (Lovy et al. 1984) and the stellar parameters in Table 2 are assumed) allows us to connect the fast photospheric variations at the base of the wind with the long wind modulation periods of days. Admittedly, the derived beat period of 6.8 days, which is observed in photosphere and base of the wind does not directly match the 1.2 and 2.4-day periods, which were derived for the periodic wind variability, but is more consistent with the longer 5.5 or 11-day repetition time scales as seen for the slow discrete absorption components (DACs) in the IUE MEGA campaign data sets. In particular the simultaneous presence of the 2.4-day period on one side and the wind emission features following the slower beat pattern on the other side and the lack of an obvious link between the two periodicities inhibits a simple unified picture

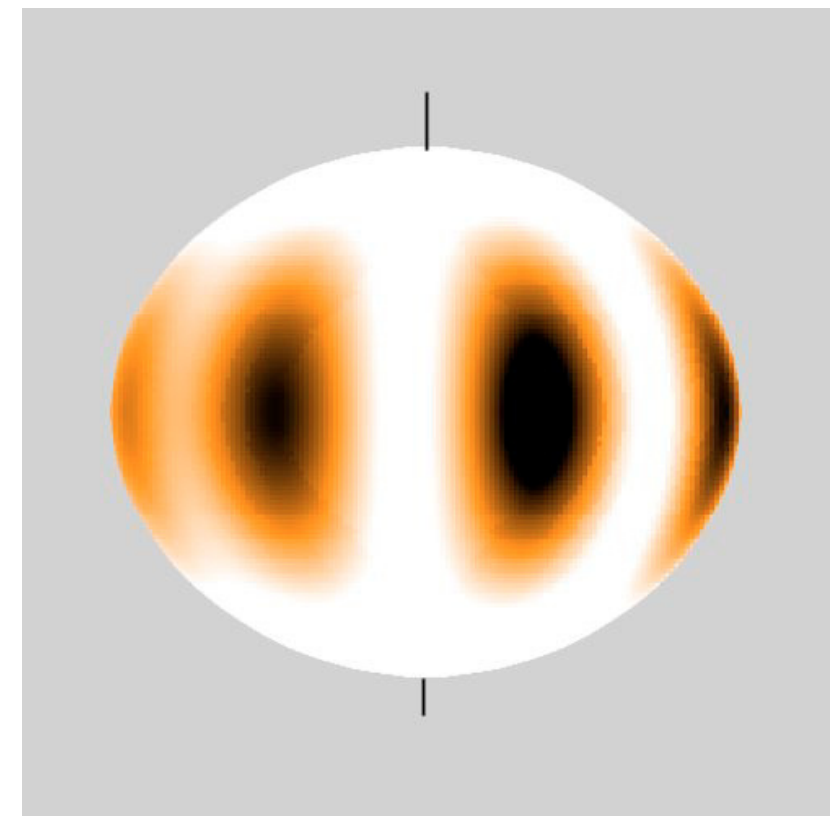

Fig. 21. Temperature distribution on the stellar surface due to NRP beat patterns (Model 1) as seen from the equatorial plane at $t=48 \mathrm{~h}$. The minimum and maximum temperatures in the visible regions are $T=21500 \mathrm{~K}$ (black) and $T=25000 \mathrm{~K}$ (white).

of the photosphere - wind connection in HD 64760. Therefore, it appears particularly important to better understand the actual physical processes, which can connect photosphere structure to stellar wind structure. Without such an understanding it will not be possible to assess the significance of any observed and derived photosphere- and wind-variability periods.

Figure 21 illustrates the spatial temperature structures in the photosphere created by the beating of the dominant two periods in our NRP Model 1. The model predicts two diametrically opposite regions of maximum beat amplitudes (constructive interference) with temperatures ranging from $T=21500 \mathrm{~K}$ to $T=25000 \mathrm{~K}$ at the stellar equator. In the regions of destructive interference 90 degrees away in azimuth, the temperature amplitudes are reduced and range from $22300 \mathrm{~K}$ to $23800 \mathrm{~K}$. In our simplified scenario these NRP-induced spatial structures locally change the lower boundary conditions of the stellar wind, which in turn causes localized structural changes in the wind. (Note, that we used a simple 2 bright -2 dark spot geometry to represent the regions of constructive and destructive interference of NRP modes).

The creation of wind structure by variation of lower boundary conditions have been investigated with hydrodynamical simulations by Cranmer \& Owocki (1996) who confirmed the formation of spiral structures (co-rotating interaction regions, CIR) originating from the collision of fast and slow streams rooted in the stellar surface. These wind structures modulate the observed stellar wind profiles as they are dragged through the line of sight to a distant observer by the underlying rotation of the stellar wind.

In contrast to earlier models for the localized regions on the stellar surface of HD 64760, we investigated the effects of the observed and predicted retrograde motion of the regions 
of maximum beat amplitudes (equal to the regions causing the variation of the lower wind boundary condition) on the geometry of the spiral structures and the resulting H $\alpha$ LPVs. Despite the fact that our simplistic $\mathrm{H} \alpha$ model does not include any hydrodynamics but is mostly geometrical, some key characteristics of the observed $\mathrm{H} \alpha$ variations could be reproduced to some extent, which was not possible with models with the surface structures locked to the stellar rotation: the relevant time scales, and the velocity evolution of the pseudo-emission and absorption features.

The crucial question to be answered is whether the NRPinduced variations of the physical parameters like local temperature, gravity, and velocity fields are actually sufficiently large to introduce significant variations at the base of the wind to build up wind structures. Cranmer \& Owocki (1996) define the spot brightness amplitude $A$ (with their $T_{0}$ corresponding to our $\left.T_{\text {equator }}\right)$ as:

$A=\left(T_{\text {spot }}^{4}-T_{\text {equator }}^{4}\right) / T_{\text {equator }}^{4}$.

Using the "spot" temperatures from our NRP Model 1, we obtain amplitudes of

$$
\begin{aligned}
A^{+} & =\left((25000 \mathrm{~K})^{4}-(23300 \mathrm{~K})^{4}\right) /(23300 \mathrm{~K})^{4} \\
& =+0.325 \\
A^{-} & =\left((21500 \mathrm{~K})^{4}-(23300 \mathrm{~K})^{4}\right) /(23300 \mathrm{~K})^{4} \\
& =-0.275
\end{aligned}
$$

which are comparable to the $A= \pm 0.5$ standard spot model used by Cranmer \& Owocki (1996) to model the IUE wind profile modulations of HD 64760 with CIRs. The azimuthal extension of the standard spot model is with $\phi=20$ deg comparable to the azimuthal extension of the bright and dark sectors of our NRP Model 1. For the same model but with the temperature effects reduced to $50 \%$ to account for non-adiabatic effects (cf. Sect. 5.4) we obtain spot brightness amplitudes of $A^{+}=0.164$ and $A^{-}=-0.146$ - according to Cranmer \& Owocki (1996) still sufficiently large to yield visible wind structure.

While NRPs are not obviously very efficient in providing localized bright spots on the stellar surface for "Radiational Driven Orbital Mass Ejection" (RDOME) or "Pulsational Driven Orbital Mass Ejection" (PDOME) in the context of the creation of Be-star disks (Owocki 2004), they might be the source of the structured winds of hot supergiants like HD 64760 as we have tried to demonstrate in this work.

However, a hydrodynamical modeling, which takes fully into account the geometrical and physical boundary conditions as created by multi-periodic NRPs in the photosphere (as e.g. shown for the simple $l=-m$ case in Fig. 21) and which further includes the retrograde motion of the beat pattern with respect to the stellar rotation is still urgently needed to provide a consistent and time dependent picture of the stellar photosphereto-wind structure of HD 64760.

Acknowledgements. O.S. wants to thank the ESO Visiting Scientist Program for the support of this project and the ESO Vitacura Science Office for its hospitality during a extended visit where a major part of this work was done.

\section{References}

Baade, D. 1984, in Liege International Astrophysical Colloquia, 115 Baade, D., \& Ferlet, R. 1984, A\&A, 140, 72

Baschek, B., Holweger, H., \& Traving, G. 1966, Astronomische Abhandlungen der Hamburger Sternwarte, 8, 26

Buta, R. J., \& Smith, M. A. 1979, ApJ, 232, 213

Cranmer, S. R., \& Owocki, S. P. 1996, ApJ, 462, 469

Fullerton, A. W., Massa, D. L., Prinja, R. K., Owocki, S. P., \& Cranmer, S. R. 1997, A\&A, 327, 699

Harries, T. J. 2000, MNRAS, 315, 722

Henrichs, H. F. 1999, LNP Vol. 523, IAU Coll. 169, Variable and Nonspherical Stellar Winds in Luminous Hot Stars, 305

Hoffleit, D., \& Jaschek, C. 1982, The Bright Star Catalogue (The Bright Star Catalogue, New Haven: Yale University Observatory (4th edition), 1982)

Howarth, I. D., Townsend, R. H. D., Clayton, M. J., et al. 1998, MNRAS, 296, 949

Kaufer, A., Prinja, R. K., \& Stahl, O. 2002, A\&A, 382, 1032

Kaufer, A., Stahl, O., Tubbesing, S., et al. 2000, in Optical and IR Telescope Instrumentation and Detectors, ed. M. Iye, \& A. F. Moorwood, Proc. SPIE, 4008, 459

Kaufer, A., Stahl, O., Wolf, B., et al. 1997, A\&A, 320, 273

Kawaler, S. D., Sekii, T., \& Gough, D. 1999, ApJ, 516, 349

Kurucz, R. 1993, ATLAS9 Stellar Atmosphere Programs and 2 km/s grid. Kurucz CD-ROM No. 13. Cambridge, Mass.: Smithsonian Astrophysical Observatory, 13

Lovy, D., Maeder, A., Noels, A., \& Gabriel, M. 1984, A\&A, 133, 307

Morel, T., Marchenko, S. V., Pati, A. K., et al. 2004, MNRAS, 351, 552

Owocki, S. P. 2004, in IAU Symp., 215, 515

Petrenz, P., \& Puls, J. 1996, A\&A, 312, 195

Prinja, R. K., Massa, D., \& Fullerton, A. W. 1995, ApJ, 452, L61

Prinja, R. K., Massa, D., \& Fullerton, A. W. 2002, A\&A, 388, 587

Schwarz, U. J. 1978, A\&A, 65, 345

Schwarzenberg-Czerny, A. 1996, ApJ, 460, L107

Stahl, O., Kaufer, A., \& Tubbesing, S. 1999, in ASP Conf. Ser., 188, Optical and Infrared Spectroscopy of Circumstellar Matter, 331

Townsend, R. H. D. 1997a, Ph.D. Thesis

Townsend, R. H. D. 1997b, MNRAS, 284, 839

van Leeuwen, F., Evans, D. W., Grenon, M., et al. 1997, A\&A, 323, L61 